\title{
树枝状纤维形二氧化硅纳米粒子的研究进展
}

\author{
王亚斌 ${ }^{1}$, 刘 忠 ${ }^{2,3}$, 史时辉 ${ }^{1}$, 呼科科 ${ }^{1}$, 张琰图 ${ }^{1}$, 郭 敏 ${ }^{2,3}$
}

(1. 延安大学 化学与化工学院, 延安 $716000 ; 2$. 中国科学院 青海盐湖研究所, 中国科学院盐湖资源综合高效利 用重点实验室, 西宁 810008; 3. 青海省盐湖资源化学重点实验室, 西宁 810008)

摘 要: 与传统二氧化硅介孔材料相比, 树枝状纤维形二氧化硅纳米粒子(Dendritic Fibrous Nano-silica, DFNS), 特 别是具备三维中心辐射状孔道和多级孔结构的球形 DFNS 拥有较高的比表面积、较大的孔体积、较高的孔渗透性 和粒子内表面更易接触性等优点。客体物质(如极小的纳米粒子)能够沿着中心辐射状孔道进行负载和/或输送, 甚至 与化学改性所得内部活性位点发生反应。因此, DFNS 是一种富有前景的载体平台, 可以用来构筑新型纳米催化剂、 吸附剂、基因/蛋白质/药物的递送系统等。大量研究表明: 球形 DFNS 与生俱来的结构优势使其能够作为 MCM-41 和 SBA-15 的理想替代材料。但是, DFNS 领域依旧存在很多需要探讨的问题。因此, 本文主要归纳分析 DFNS 的 结构特征、常用结构模型、新型结构和实时应用。希望能够给予材料和化学科学家一些参考, 促进 DFNS 的蓬勃 发展。

关 键 词：树枝状纤维形; 二氧化硅; 结构模型; 新颖结构; 实时应用

中图分类号: O611; TB383 文献标识码: A

\section{Research Progress of Dendritic Fibrous Nano-silica (DFNS)}

\author{
WANG Ya-Bin ${ }^{1}$, LIU Zhong ${ }^{2,3}$, SHI Shi-Hui ${ }^{1}$, HU Ke-Ke ${ }^{1}$, ZHANG Yan-Tu ${ }^{1}$, GUO Min ${ }^{2,3}$ \\ (1. College of Chemistry and Chemical Engineering, Yan'an University, Yan'an 716000, China; 2. Key Laboratory of Compre- \\ hensive and Highly Efficient Utilization of Salt Lake Resources, Qinghai Institute of Salt Lakes, Chinese Academy of Sciences, \\ Xining 810008, China; 3. Key Laboratory of Salt Lake Resources Chemistry of Qinghai Province, Xining 810008, China)
}

\begin{abstract}
Dendritic fibrous nano-silica (DFNS), especially the sphere-shaped with three-dimensional (3D) centerradial channels and hierarchical pores, possess higher specific surface areas, larger pore volumes, higher pore permeability, more accessible internal spaces, etc. Guest substances (e.g., ultrasmall nanoparticles) can be loaded onto and transported in the radial nanochannels, or can even react with the chemically active sites in these nanochannels. As a result, DFNS can serve as promising platforms to construct novel nanocatalysts, adsorbent materials, and delivery systems for genes, proteins or drugs. A majority of investigations about DFNS have demonstrated that silica nanospheres with this special topography have inherent superiorities over traditional mesoporous MCM-41 or SBA-15, and can be perfect alternatives. Nevertheless, reviews on DFNS are limited, and there still exist plenty of issues that need to be probed into. Therefore, this comprehensive review provides a critical survey on DFNS' structural characteristics, commonly used structural models, novel structures, real-time applications, etc. We sincerely expect that this paper
\end{abstract}

收稿日期: 2018-05-29; 收到修改稿日期：2018-06-29

基金项目: 国家自然科学基金(U1607105); 中国科学院青年促进会项目(2016377); 陕西省自然科学基金(2016ZDJC-19); 延安 大学博士科研启动项目(YDBK2017-39, YDBK2016-14); 青海省科技项目(2018-GX-101, 2018-ZJ-722)

National Natural Science Foundation of China (U1607105); Youth Innovation Promotion Association (2016377); Natural Science Foundation of Shaanxi Province (2016ZDJC-19); Doctoral Research Program of Yan'an University (YDBK2017-39, YDBK2016-14); Science and Technology Funding in Qinghai Province (2018-GX-101, 2018-ZJ-722)

作者简介: 王亚斌(1985-), 男，博士，讲师. E-mail: ybw@yau.edu.cn

通讯作者: 刘 忠, 副研究员. E-mail: liuzhong@isl.ac.cn 
could give material scientists and chemists certain inspiration to accelerate DFNS family's booming evolution.

Key words: dendritic fibrous; silica; structural models; novel structures; real-time applications

介孔材料一直以来受到广泛关注，归因于其特 殊的物理化学性能，如比表面积大、介孔性高、机 械性能优异等 ${ }^{[1]}$ 。与同等尺寸的无孔实心结构材料 相比，多级孔结构或复杂多层级结构使得介孔材料 性能提升，作为载体有广阔的前景，适用于催化、选 择性吸附和二氧化碳捕捉等科学领域 ${ }^{[2]}$ 。自 Mobil 公司于 1992 年开创性地制备 MCM-41 之后 ${ }^{[3]}$, 大量 工作专注于介孔材料的理论研究、制备策略、表征 方法和新兴应用领域。由赵东元等 ${ }^{[4]}$ 于 1998 年合成 的 SBA-15 将二氧化硅介孔材料发展推进到新的高 潮。此后，尽管众多课题组开展大量工作以制备新 型二氧化硅介孔材料, 但研发过程困难重重、成果 不多。

直到 2010 年, Polshettiwar 等 ${ }^{[5]}$ 使用微波反应器, 通过简单的乳液法里程碑式地制备了树枝状纤维形 二氧化硅纳米球, 命名为 $\mathrm{KCC}-1$ (类似于 MCM-41 命名规则)。KCC-1 为 KAUST Catalysis Center 的首 字母缩写, 而 KAUST 代表 King Abdullah University of Science and Technology。不同反应条件可以 调控 DFNS 内部介孔尺寸 $(2 \sim 30 \mathrm{~nm})$ 和纳米球直径尺 寸 $(170 \sim 1120 \mathrm{~nm})^{[6]}$ 。因此, KCC-1 这一称谓及树枝 状纤维形纳米球被大多数研究人员认识、接受和使 用 ${ }^{[7-14]}$ 。同年，杜傘课题组 ${ }^{[15]}$ 采用乙醚乳液体系制 备了树枝状纤维形二氧化硅纳米球, 产物被命名为 Hierarchical Mesoporous Silica Nanoparticles (HMSNs), 对该类结构纳米粒子的发展做出了巨大贡献 ${ }^{[16-21]}$ 。
此后，具有“树枝状纤维形”结构特征的纳米粒子开 始蓬勃发展, 从单一简单结构进化为多级复杂结构, 应用范围也日益增大。2014 年, 赵东元院士课题组 ${ }^{[22]}$ 采用油水双相分层法制备了层级结构可控的多代 DFNS, 命名为 Three dimensional dendritic mesoporous silica nanospheres (3D dendritic MSNSs)。所谓的 多代层级结构可控是指每个壳层都具有相同的孔径 尺寸，而壳层与壳层之间具有不同的孔径尺寸。

图 1 为本课题组通过水热反应釜制备所得具有 树枝状纤维形结构二氧化硅纳米球的透射电子显微 镜(TEM, (a) (c))和扫描电子显微镜(SEM, (d) (f))照 片 $^{[23]}$ 。照片显示单分散树枝状纤维形二氧化硅纳米 球由沿着粒子中心到表面辐射方向排列的纳米褶皱 组成。中心辐射状孔道相应地在纳米禇皱之间形成, 其孔径尺寸从粒子内部到外部逐渐增大。由褶皱挤 压形成的多级孔不均匀散布于二氧化硅球表面, 随机测量的褶皱片厚度分布在 $8.3 \sim 14.2 \mathrm{~nm}$ 之间 (图 1(e)), 不规则多级孔的孔径在 10.9 35.2 nm 范围 之间(图 1(f))。杜金和 Polshettiwar 课题组分别于 2015 年 ${ }^{[16]}$ 、2016 年 ${ }^{[24]}$ 和 2017 年 ${ }^{[25]}$ 对 DFNS 学科做 了综述，介绍了 DFNS 的合成策略及应用进展。基 于本文作者课题组和其他课题组的研究成果, 本综 述则对上述文章未涉及部分进行补充(如结构模型 分析), 对存在的一些要点进行尝试性探讨(如称谓 统一), 也对近期发表的 DFNS 内容进行更新(新兴 结构及实时应用)。
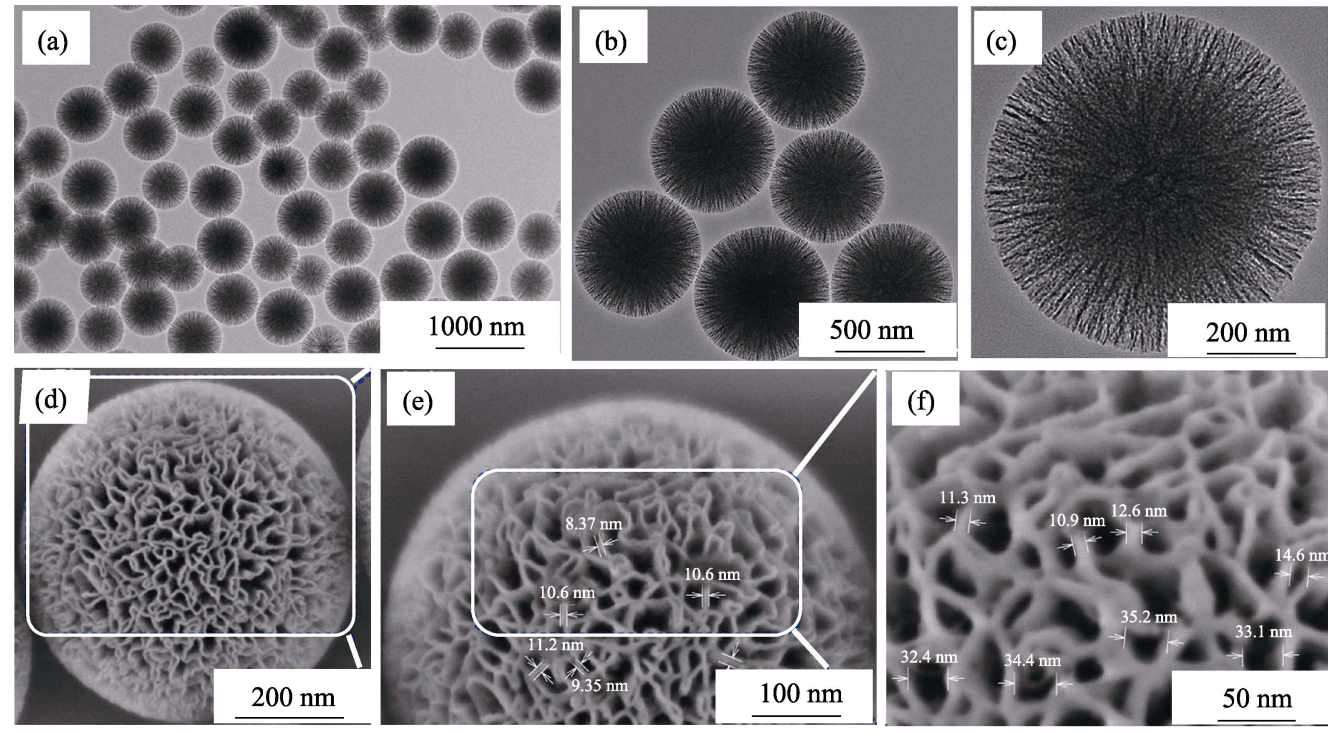

图 1 树枝状纤维形二氧化硅纳米球的透射电镜(a) (c)和扫描电镜(d) (f)照片 ${ }^{[23]}$

Fig. 1 TEM (a-c) and SEM (d-f) images of silica-based nanospheres with dendritic fibrous morphologies ${ }^{[23]}$ 


\section{1 称谓概述}

\section{1 各类称谓总结}

随着学者们对树枝状纤维形二氧化硅纳米球的 深入研究, 众多称谓依次出现, 导致了名称不统一 现象。但是，这些称谓基本都在描述具有中心辐射 状孔道和多级孔结构的 DFNS。表 1 归纳了常见的 英文称谓、英文称谓缩写及中文名。由表可知: (1) “树 枝状”和“纤维形”这两类修饰词使用较多，“禇皱状” 修饰词偶尔出现; (2)部分称谓不能明确描述对象的 具体结构。例如，当英文称谓缩写为 DMSNs、WMS 和 FSNs 时, 不易看出所指对象为二氧化硅纳米球 还是非球形纳米粒子; (3)部分称谓不能明确界定 DFNS 为纳米粒子还是微米粒子。笔者根据以上现 象推断, 正是鉴于这些原因, Polshettiwar 课题 组 ${ }^{[25-29]}$ 于 2017 年重新命名具有该类结构的纳米粒 子为 DFNS。考虑到 Polshettiwar 课题组对 DFNS 纳 米粒子的巨大贡献和影响力, 本综述也遵循此命名 称谓规范。需要对 DFNS 称谓作出如下说明: (1)笔 者表述具有中心辐射状孔道和多级孔结构的 DFNS 为纳米粒子而不是纳米球。因为随着对具有该类结 构纳米粒子的深入研究, 非球形结构如羽毛球形 ${ }^{[48]}$ 和椭球形 ${ }^{[80]}$ 被相继开发出来。此外, 英文称谓 “Dendritic fibrous nano-silica (树枝状纤维形二氧化硅纳 米粒子)”和“Dendritic fibrous nano-spheres (树枝状纤维 形二氧化硅纳米球)”的首字母缩写均为 DFNS, 这会引
起描述对象的不确定性。(2)“介孔”一词(Mesoporous) 也没有出现在 DFNS 中, 因为绝大多数 DFNS 的孔 径或 DFNS 多级孔中的绝大部分均处在 2 50 nm 之

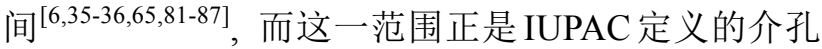
尺寸范围 ${ }^{[88]}$ 。(3)由图 1 可知, TEM 能够展示纳米粒 子总体形貌和结构特征(中心辐射状), 而 SEM 重在 揭示细节结构特征(纳米褶皱)。DFNS 称谓中的“树 枝状(Dendritic)”和“纤维形(Fibrous)”很大程度衍生 自 TEM 照片。

\section{2 对 “树枝状” 与 “纤维形” 的理解}

首先，修饰词“树枝状(dendritic)”最先被杜金课 题组 ${ }^{[89]}$ 所采用，用来描述 DFNS 的结构特征(图 2(a)), 继而才被 Polshettiwar 课题组(图 2(b) $)^{[11]}$, 赵 东元课题组 ${ }^{[22,31-32]}$, 及其他课题组采用 ${ }^{[34-35,90]}$ 。需要 特别强调的是，杜金课题组使用“树枝状(dendritic)” 这一词语, 则是受到树枝状有机聚合物大分子的启 发。该类大分子通常呈球形, 并具有三维分支构造 $(\text { 图 2(d) })^{[16-17]}$, 每一次分支代表一次迭代, 而每一次 迭代步骤被称为一代(Generation, G) ${ }^{[16,91]}$ 。图 2(d) 代表了树枝状大分子结构代数 $\mathrm{G}$ 由 0 到 4 的逐步增 迭过程。当第 4 代重叠在一起, 其形成的二维结构 则相似于 TEM 所显示出的结构形貌。其次, 大多数 树枝状纤维形二氧化硅纳米球遵循图 2(b)的形貌，兼 备树枝状和纤维形特征。可以推测, 当中心辐射状 孔道开口极大的时候, 球形 DFNS 呈“树枝状态” (图 2(a)); 当中心辐射状孔道开口极其微小的时候,

表 1 树枝状纤维形二氧化硅纳米粒子称谓概括

Table 1 English and Chinese appellations, and their abbreviations for silica nanoparticles with dendritic fibrous structures

\begin{tabular}{|c|c|c|c|c|}
\hline No. & English appellation & $\begin{array}{l}\text { Appellation } \\
\text { abbreviation }\end{array}$ & $\begin{array}{l}\text { Chinese } \\
\text { appellation }\end{array}$ & Ref. \\
\hline 1 & Dendritic fibrous nanosilica & DFNS or (KCC-1) & 树枝状纤维形二氧化硅纳米粒子 & {$[25-28,30]$} \\
\hline 2 & Three dimensional dendritic mesoporous silica nanospheres & 3D dendritic MSNSs & 三维树枝状介孔二氧化硅纳米球 & {$[22,31-32]$} \\
\hline 3 & Dendrimer-like silica nanoparticles with hierarchical pores & HPSNs & 树枝状多级孔二氧化硅纳米粒子 & {$[17,21,33-34]$} \\
\hline 4 & Dendritic mesoporous silica nanoparticles & DMSNs & 树枝状介孔二氧化硅纳米粒子 & [35-42] \\
\hline 5 & Dendritic mesoporous silica nanospheres & DMSNs & 树枝状介孔二氧化硅纳米球 & [43-45] \\
\hline 6 & Fibrous mesoporous silica microspheres & FMSMs & 纤维形介孔二氧化硅微球 & [46-47] \\
\hline 7 & Wrinkled silica nanoparticles & WSNs & 褶皱状二氧化硅纳米粒子 & [48-52] \\
\hline 8 & Wrinkled mesoporous silica & WMS & 褶皱状介孔二氧化硅 & {$[53-56]$} \\
\hline 9 & Wrinkled mesoporous silica nanoparticles & WMSNs & 褶皱状介孔二氧化硅纳米粒子 & {$[57-58]$} \\
\hline 10 & Radial-like mesoporous silica & RMS & 中心辐射状介孔二氧化硅 & [59-60] \\
\hline 11 & Fibrous silicon dioxides spheres & FSS & 纤维形二氧化硅球 & [61] \\
\hline 12 & Fibrous silica nanoparticles or nanospheres & FSNs & 纤维形二氧化硅纳米粒子或纳米球 & [62] \\
\hline 13 & Hierarchically and radially mesoporous silica & HRM & 分层次和辐射状介孔二氧化硅 & {$[63]$} \\
\hline 14 & Hierarchically structured spherical mesoporous nanoflowers & HSMNF & 分层次结构球形介孔纳米花” 和 & [64] \\
\hline 15 & Mesostructured silica nanoparticles & MSNs & 介孔结构二氧化硅纳米粒子 & {$[33,65-67]$} \\
\hline 16 & "Dendritic" & - & & {$[13,68-70]$} \\
\hline 17 & "Fibrous" & - & & [71-79] \\
\hline
\end{tabular}




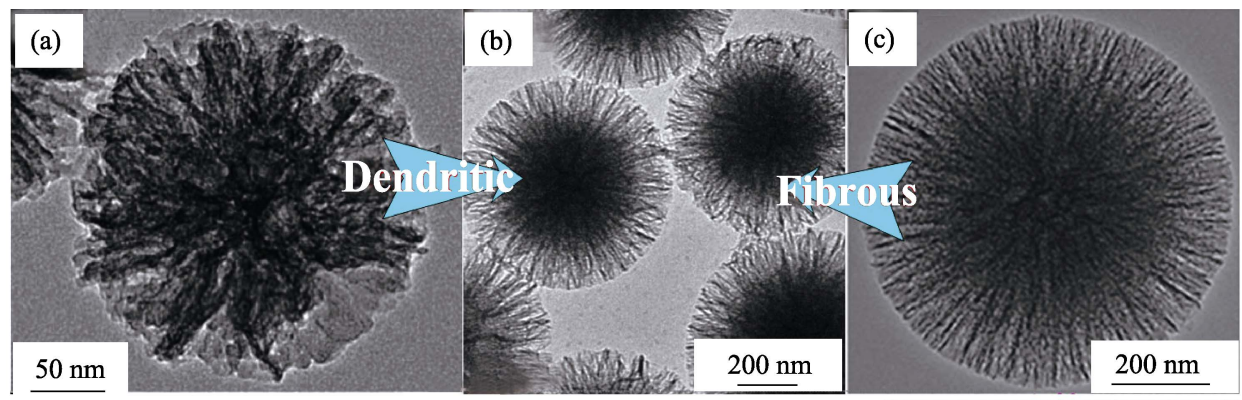

(d)
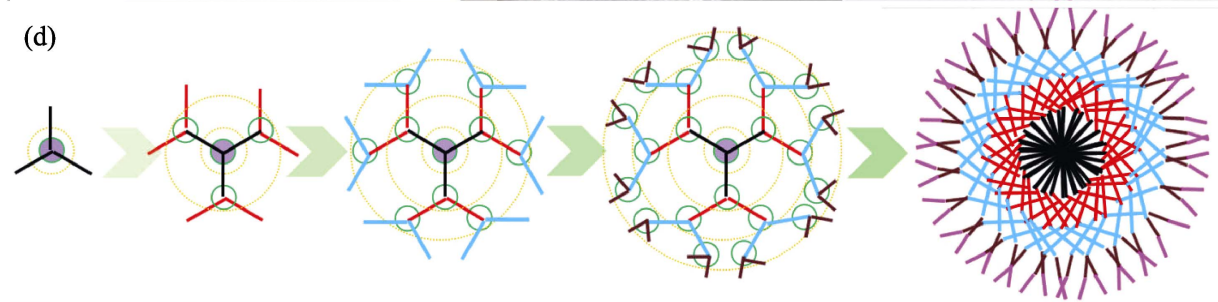

图 2 树枝状纤维形二氧化硅纳米球的结构态转变 ${ }^{[89]}$

Fig. 2 The structural transformation of a DFNS from a quasi dendritic state ${ }^{[89]}$ (a) to a quasi radially fibrous state ${ }^{[23]}$ (c) through an intermediate state of "dendritic fibrous" ${ }^{\text {"[11] }}$ (b). The forming process of a dendrimer by iterative grafting steps (d)

DFNS 呈“纤维形态”(图 2(c))。因此, 没有强制要求 同时使用树枝状纤维形去描述具有此类结构的纳米 粒子, 描述性称谓需要根据实际形貌特征决定。但 是，最基本的命名原则为尽量使用“树枝状”或者 “纤维形”, 这样才能使具有三维中心辐射状和多级 孔结构的纳米粒子称谓更加规范化。随着 DFNS 复 杂结构的出现(如空心和多壳结构), 必须对该类结 构纳米粒子的称谓提前作出统一, 否则其他结构修 饰词会让复杂结构的名称更加混乱。

\section{2 研究进展}

过去五年, DFNS 越来越受到科研工作者关注 (图 3(a))。依据 ACS、Wiley、RSC、Elsevier、Springer 以及 CNKI 数据库收录文献统计, 以 DFNS 为主题 的文献总数达到约 200 篇, 包括期刊论文、会议论 文、硕士博士论文、专利等。

DFNS 文献数量逐年增加, 且在 2014 年开始爆 发式发展。截止 2018 年 4 月, 该主题发表论文总量 已将近 2017 年全年发表文献总数量的一半，依旧呈 上升趋势。DFNS 应用领域极其广泛(图 3(b)), 包括 催化 ${ }^{[7,9-10,14,30,38,44,49,50,54-55,63,82,86,89-90,92-116]}$ ，癌症和 肿瘤治疗 ${ }^{[34,40,47,56-58,84,117-118]}$, 药物递送 ${ }^{[39,46,117-121]}$,

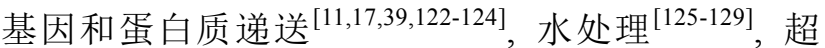

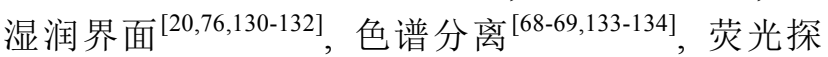
针 $^{[135-137]}$, 二氧化碳捕捉 ${ }^{[100,138-139]}$, 电池 ${ }^{[61]}$, 生物成 像 ${ }^{[31,34]}$, 储热 ${ }^{[59]}$, 光子晶体 ${ }^{[140]}$, 复合材料 ${ }^{[87]}$ 等。催 化应用领域吸引了最多关注(约占 $38 \%$ ), 生物应用
领域达到约 32\%(包括癌症和肿瘤治疗、药物递送、 基因和蛋白质递送等)，上述两个应用领域也是其 他介孔材料应用研究热点。
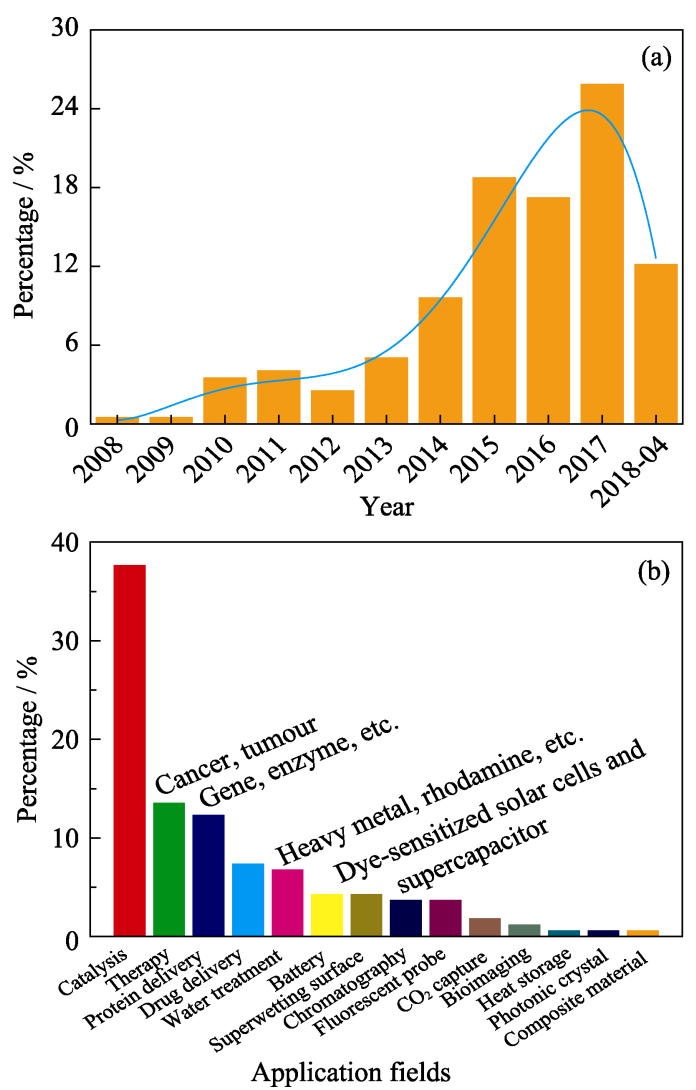

图 3 DFNS 相关文献逐年发表情况(a)及应用领域范围(b) Fig. 3 The percentages of published literatures about DFNS from 2008 to 2018 (a), and the percentages of various applications for DFNS (b) 


\section{3 结构特征}

\section{1 大孔或/和微孔的存在性}

依据 IUPAC 定义, 介孔材料分为 3 类 ${ }^{[88]}$ : 孔径 小于 $2 \mathrm{~nm}$ 的微孔材料、孔径分布于 2 50 nm 的介 孔材料、以及孔径大于 $50 \mathrm{~nm}$ 的大孔材料。DFNS 孔径大都位于 2 50 nm 之间 ${ }^{[6,35,65,81-82,85]}$, 但也有一 些球形 DFNS 中心辐射孔道尺寸超出 $50 \mathrm{~nm}$ 。例如, 杜金课题组 ${ }^{[21]}$ 成功制备了平均孔径高达 $148 \pm 45 \mathrm{~nm}$, 纳米球基体直径尺寸可控于 300 700 $\mathrm{nm}$ 的氨基官 能团修饰的球形 DFNS。Seo 等 ${ }^{[82}$ 通过引入乙酸苄 酯(BENA)来扩孔，随着 BENA 由 $5 \mathrm{~g}$ 增加到 $7 \mathrm{~g}$ 甚 至 $9 \mathrm{~g}$, 平均孔径被扩张为 $40 \mathrm{~nm}$ 至 $50 \mathrm{~nm}$, 甚至 $65 \mathrm{~nm}$ 。 由图 1 可知, 沿中心辐射方向排列的纳米褶皱是构 造 DFNS 骨架的基本结构单元，纳米禩皱的空间拓 扑排列构成了中心辐射状孔道。孔径从粒子内部到 表面逐渐增大, 可以将这些不规则孔道形容成形状 规则的圆雉形。因此微孔尺寸在纳米球中心(球核) 出现的几率最大, 而介孔尺寸大多集中在外表面附 近。需指明一点, 微孔可能不仅存在于圆锥形顶端 处(纳米球中心), 也可能镶嵌于纳米褶皱内部。即 DFNS 多级孔结构由中心辐射状多级孔道和存在于 纳米褶皱中的小尺寸介孔协同构成。

Seo 等 ${ }^{[82]}$ 指出微孔和介孔共存于球形 DFNS, 是多级孔的一种不规则和分层次组合。Zhang 等 ${ }^{[83]}$ 通过 Barrett Joyner Halenda(BJH) 方法计算出微孔和 介孔所占比例, 前者约占 $1 / 7$, 而后者约为 $1 / 3$ 。其 他研究小组先后也确定球形 DFNS 氮气吸脱附曲线 为 IV 型, 并伴有 $\mathrm{H}_{3}$ 型滞后环 ${ }^{[141-142]}$ 。依据 IUPAC 规定, $\mathrm{H}_{3}$ 型滞后环的出现起源于片状结构堆积而成 的微孔结构 ${ }^{[8]}$ 。Suendo 等 ${ }^{[12]}$ 通过 Scherrer 方程求得 镶嵌于纳米褶皱内部的微孔孔径约为 $1.2 \mathrm{~nm}$ 。

\section{2 结构和形貌模型}

需要强调一点, 本章节所指的结构模型均归属 于树枝状纤维形二氧化硅纳米球，因为到目前为止， 球形 DFNS 占该学科研究的绝大部分。树枝状纤维 形二氧化硅纳米球的模型也包括三维(3D)示意结构

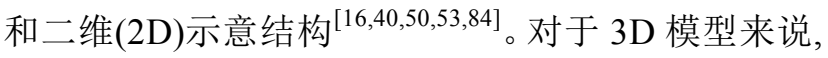
Lee 等 ${ }^{[143]}$ 给出的模型最大程度地保留了 DFNS 的结 构特点(类似于图 1(d))。本课题组给出如图 4(a)所示 的 3D 模型, 从图 4( $\left.a_{1}\right)$ 到 $\left(a_{3}\right)$, 多级孔道的孔径随纳 米褶皱间距增大而变大。另外一个常用来描绘球形 DFNS 的结构模型如图 4(b)所示 ${ }^{[17,22,44,53,144-145]}$, 可 称为理想模型。从图 $4\left(b_{1}\right)$ 到 $\left(b_{3}\right)$, 圆雉形孔径逐渐增 大。赵东元等 ${ }^{[22,146]}$ 利用图 4(b)模型描绘其课题组制 备的多层级结构可控 DFNS, 模型与实际产物形

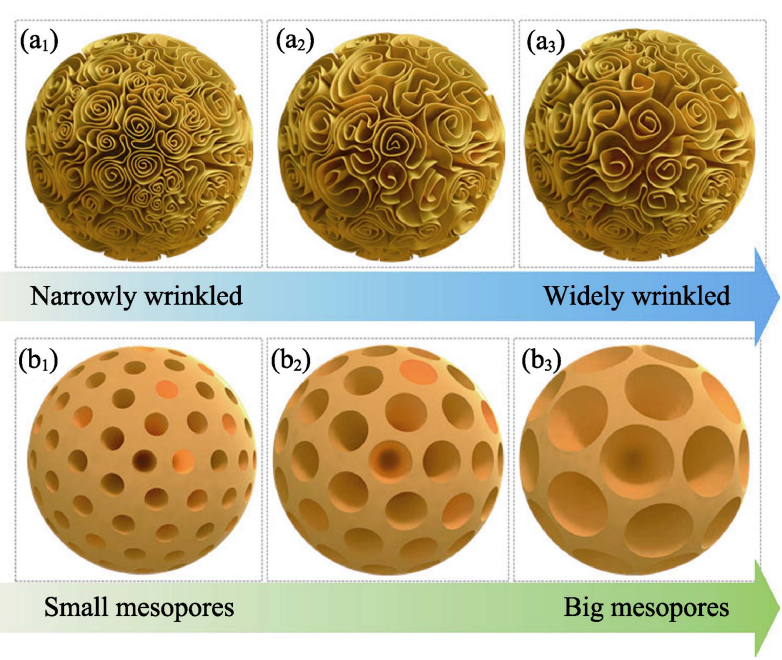

图 4 球形 DFNS 常用结构模型

Fig. 4 The commonly used morphological models for DFNS spheres.

(a) The practical and the most appropriate models, as well as (b) the ideal ones

貌贴切。但是，图 4(b)模型也被用来示意一些核一壳 状介孔硅球 ${ }^{[147]}$, 模型与实际所得产物形貌相差较 大, 实际产物的 TEM 照片观察不到树枝状结构纹 理。与真正核-壳树枝状复合纳米粒子对比，图 4(b) 模型不适用于这些研究的反应产物示意。反面理解, 通过 $\mathrm{KCC}-1$ 方式制备的以沸石 $\mathrm{TS}^{-180]}$ 、 ZSM-5 $5^{[107,148]}$ 和 $\mathrm{Fe}_{3} \mathrm{O}_{4}{ }^{[8]}$ 为核的 DFNS, 其壳均呈现 树枝状纤维形，与图 1 中的 SEM 和 TEM 结果相近。 因此, 笔者推荐读者使用图 4(a)模型来描述球形 DFNS, 图 4(b)模型需勘酌慎用, 易引起混淆。

对于 DFNS 的 2D 模型来说, 可以想象 DFNS 纳米球沿球心被切开。因此, 相应的 TEM 照片能够 反映该结构, 如图 1(c)所示。Wang 等 ${ }^{[114]}$ 使用球形 DFNS 的 TEM 照片来示意其结构, 贴切实际形貌且 简单易懂。也有课题组 ${ }^{[127]}$ 采用不同长度的实线取向 排列，绘制类似树枝状纤维形的 2D 图像来表示球 形 DFNS 的结构特征(图 5(a))。早在 2011 年, 杜金金 等 ${ }^{[149]}$ 将具有多级孔结构的二氧化硅微米球或纳米 球分为 4 类, 其中包括放射状分层次结构的介孔球 (图 5(b)) 和具有不同孔径分布的介孔球(图 5(c))。 Lin 等 ${ }^{[150]}$ 使用图 5(d) 示意复合纳米粒子 $\mathrm{Fe}_{3} \mathrm{O}_{4} @ n \mathrm{SiO}_{2} @ m \mathrm{SiO}_{2}$ 的结构, $n \mathrm{SiO}_{2}$ 和 $m \mathrm{SiO}_{2}$ 分别 代表均匀的二氧化硅层和介孔二氧化硅壳。笔者认 为更应该用图 5(c) 和磁性核的复合结构来描述 $\mathrm{Fe}_{3} \mathrm{O}_{4} @ n \mathrm{SiO}_{2} @ m \mathrm{SiO}_{2}$ (图 5(e)), 其与该研究中实际 产物的 TEM 照片完美契合。最近, Dong 等 ${ }^{[97,110]}$ 采 用图 5(f)所示的太阳花状示意图描述核一壳结构的 $\gamma-\mathrm{Fe}_{2} \mathrm{O}_{3} @ \mathrm{SiO}_{2} @ \mathrm{KCC}-1$, 本论文作者认为 $\gamma-\mathrm{Fe}_{2} \mathrm{O}_{3}$ 和 图 5(a)的组合结构更加贴切, 才能显示树枝状纤维 形纹理。 


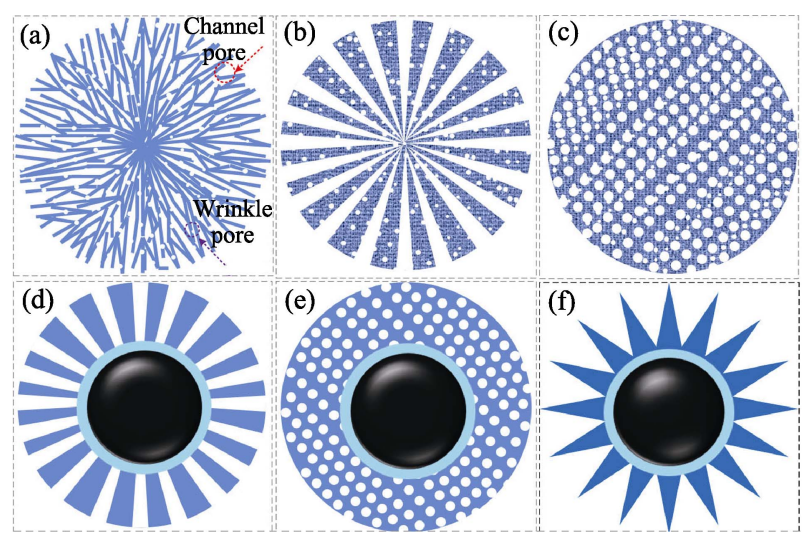

图 5 不同结构二氧化硅纳米粒子的二维示意图 ${ }^{[110,127,149-150]}$

Fig. 5 Schematic diagrams of 2D planar structures from various silica nanospheres ${ }^{[110,127,149-150]}$

(a) DFNS; (b) Hierarchical radial porous sphere; (c) Hierarchical porous sphere; (d) Radial porous sphere with a magnetic core; (e) Porous sphere with a magnetic core; (f) Sunflower-like magnetic porous sphere

\section{4 合成方法}

\section{1 经典合成方法的适用性}

DFNS 的合成策略及应用领域在前述的三篇综 述已有详尽报道 ${ }^{[16,24-25]}$, 读者可自行查阅, 本文主 要侧重分析合成方法的优越性和实时进展。表 2 对 比分析了由 Polshettiwar、杜金和赵东元等开发的 KCC-1 环己烷乳液法、乙醚乳液法和油水双相分层 法的优越性。经本课题组重复试验, 上述 3 种方法 均能成功制备球形 DFNS。然而油水双相分层法需 要更加细致的操作, 这使得该方法操作过程更具规 范性 (与其合成机理有关，参见文献 [22])。尽管 KCC-1 环己烷乳液法和乙醚乳液法制备的球形 DFNS 粒径范围均可控于 100 1300 nm 范围, 但是 一锅制备出的终产物粒径分布不均匀 ${ }^{[5,10,17,21,109]}$,
而油水双相分层法制备的纳米粒子粒径十分均匀 ${ }^{[22]}$ 。 从 $\mathrm{KCC}-1$ 环己烷乳液法到乙醚乳液法, 存在于 DFNS 表面的孔逐渐变得不规则, 这是因为不同体 积的乙醚冲破乳液滴, 形成极其不均匀的孔道 ${ }^{[15,17,21]}$ 。 只在乙醚乳液法产物中观察到了副产物 ${ }^{[21]}$, 包括 $1 \sim 10 \mu \mathrm{m}$ 的空心介孔球、1 $2 \mu \mathrm{m}$ 空心介孔球和具有 多级孔的空心介孔球(这些副产物可以通过逐级离 心分离法去除)。

综上所述，每一种方法具有自身优势，如乙醚 乳液法能够制备孔径尺寸极大的介孔，有利于大分 子和大尺寸纳米粒子负载于 DFNS 孔道内。 $\mathrm{KCC}-1$ 环己烷乳液法和乙醚乳液法均能得到生长不成熟的 中间产物，油水双相分层法没有中间产物的形成。 为了制备球形 DFNS, KCC-1 衍生的乳液制备方法 被广泛采用 $(\sim 70 \%)$, 接着是乙醚微乳液法 $(\sim 17 \%)$, 最后是油水双相分层法( $3 \%)$ 。大量研究亟需投入到 乙醚乳液法反应体系和油水双相分层法中去。

\section{2 其他合成方法概述}

基于强抗衡离子机理, Zhang 等 ${ }^{[81]}$ 在温和反应 条件下使用溶胶一凝胶技术通过软模板法成功制备 了公斤级的单分散球状 DFNS, 粒子尺寸集中在 100 150 nm, 纳米禇皱的厚度偏大, 约为 10 20 nm。 该课题组利用嵌段共聚物(聚环氧乙烷 106 - 聚环氧丙 烷 70-聚环氧乙烷 106 , Pluronic F127)作为粒子增长抑 制剂, 不同烷基链长度的咪唑鎓盐离子液体作为助 表面活性剂，制备了金原子掺杂的复合纳米粒子 Au\&DFNS，粒子尺寸在 50 300 nm 范围可控 ${ }^{[35]}$ 。

最近, 该课题组采用十二烷基苯磺酸钠(SDS) 和溴代十六烷基三甲基铵(CTAB)为双模板，制备了公 斤级的球形 DFNS, 粒径约为 $100 \mathrm{~nm}^{[151]}$ 。Guo 等 ${ }^{[152]}$ 用醛类化合物代替乙醚, 制备了介孔结构可调和

表 2 KCC-1 环己烷乳液法、乙醚乳液法和油水双相分层法适用性对比

Table 2 Comparison of the approaches applied to synthesize DFNS spheres, including: cyclohexane emulsions for KCC-1, ethyl ether emulsion for HSMNs, and biphase stratification technique for 3D dendritic MSNSs

\begin{tabular}{cccc}
\hline Synthetic approach & $\begin{array}{c}\text { Cyclohexane emulsions } \\
\text { for DFNS or KCC-1 }\end{array}$ & $\begin{array}{c}\text { Ethyl ether emulsion } \\
\text { for HSMNs }\end{array}$ & $\begin{array}{c}\text { Biphase stratification } \\
\text { for 3D dendritic MSNSs }\end{array}$ \\
\hline Pore size range & $c a .2-30 \mathrm{~nm}$ & $c a .8-200 \mathrm{~nm}$ & $c a .2 .8-10 \mathrm{~nm}$ \\
Particle size range & $c a .170-1120 \mathrm{~nm}$ & $c a .100-1100 \mathrm{~nm}$ & $c a .180-280 \mathrm{~nm}$ \\
Repeatability & Excellent & Excellent & Excellent \\
Maneuverability & Easy & Easy & Normal \\
Particles'uniformity & Poor & Poor & Excellent \\
Pores' uniformity & Good & Average & None \\
By-product & None & Some & None \\
Intermediate products & Some & Some & $\sim 3 \%$ \\
Universality & $\sim 70 \%$ & $\sim 17 \%$ & Uniformity for fine control \\
Significance & Facility and universality & Macropores for diverse guests &
\end{tabular}


尺寸可控的球形 DFNS。当短链的乙醛被用作共溶 剂, DFNS 粒子尺寸能够通过改变反应液 $\mathrm{pH}$ 和乙醛 用量来控制在 40 850 nm 范围内。当长链的丙醛或 丁醛被使用时, DFNS 形貌尺寸小于 $130 \mathrm{~nm}$, 但辐 射状介孔开口变大且大量小孔嵌在其中, 形成多级 孔结构。

\section{5 新兴结构}

\section{1 核一壳结构}

\subsection{1 磁性核-壳结构}

Liu 等 ${ }^{[8]}$ 成功制备了单分散磁性核一壳状 $\mathrm{Fe}_{3} \mathrm{O}_{4} @ \mathrm{SiO}_{2} @ \mathrm{KCC}-1$ 。首先, $170 \mathrm{~nm}$ 的 $\mathrm{Fe}_{3} \mathrm{O}_{4}$ 磁性 核通过溶胶一凝胶法包覆一层非介孔二氧化硅层。然 后, 通过静电作用将溴化十六烷基吡啶(CPB) 自组 装在 $\mathrm{Fe}_{3} \mathrm{O}_{4} @ \mathrm{SiO}_{2}$ 表面, 形成表面活性剂模板胶 体。最后, 通过 TEOS 的水解缩合以及模板剂的去 除, 形成了如图 6(a)所示的复合纳米球。直径约 $320 \mathrm{~nm}$ 的 $\mathrm{Fe}_{3} \mathrm{O}_{4} @ \mathrm{SiO}_{2} @ \mathrm{KCC}-1$ 被用来吸附亚甲基蓝, 饱和 吸附量为 $48.0 \mathrm{mg} / \mathrm{g}$ 。动力学吸附实验表明: 在最初 震荡处理 $5 \mathrm{~min}$ 内，吸附能力达到 $78 \%$ ，且在 $30 \mathrm{~min}$ 内达到吸附平衡, 中心辐射状孔道和多级孔结构加 速了吸附速度。Sadeghzadeh 等 ${ }^{[153]}$ 则首先用 3-氯丙 基三乙氧基硅烷对 $\mathrm{Fe}_{3} \mathrm{O}_{4} @ \mathrm{SiO}_{2} @ \mathrm{KCC}-1$ 进行化学 改性, 然后将氯功能化的复合纳米粒子浸入芐基四 氮唑、正丁基锂和氯金酸溶液。所得磁性 $\mathrm{Fe}_{3} \mathrm{O}_{4} @ \mathrm{SiO}_{2} @ \mathrm{KCC}-1 \& \mathrm{Au}$ 能够高选择性环化 $\mathrm{CO}_{2}$ 和炔丙基胺, 高产率生成恶唑烷酮。Piao 等 ${ }^{[154]}$ 将 $5 \sim 10 \mathrm{~nm}$ 的氧化铁纳米晶自组装为 $280 \pm 37 \mathrm{~nm}$ 树莓 状磁性核(Superparamagnetic Iron Oxide Nanocluster, SION), 在其表面制备 DFNS 壳, 生成 $317 \pm 56 \mathrm{~nm}$ 的 复合纳米粒子 SION@DFNS。将 $\mathrm{TiO}_{2}$ 纳米颗粒载入 树枝状纤维形孔道内, 形成 SION@DFNS/TiO 2 催化 剂。相对于商业化的二氧化钛 P25 粒子, 该复合催 化剂展现了更快的染料降解能力。Zhang 等 ${ }^{[13]}$ 制备 了磁性 $\mathrm{Fe}_{3} \mathrm{O}_{4} @ \mathrm{KCC}-1$, 将脂肪分解酵素(Candida Rugosa Lipase, CRL)负载于该多级结构。28 d 后, 所 得 $\mathrm{Fe}_{3} \mathrm{O}_{4} @(\mathrm{KCC}-1) / \mathrm{CRL}$ 结构中的脂肪分解酵素活 性保持为 $89 \%$, 而没有负载于 $\mathrm{Fe}_{3} \mathrm{O}_{4} @ \mathrm{KCC}-1$ 的 CRL 活性仅为 $25 \%$ 。赵东元等 ${ }^{[144]}$ 开发了一种剪切 力辅助的双液界面共自组装方法来合成磁性核一壳 DFNS 复合纳米粒子。首先, 在水溶性的 $\mathrm{Fe}_{3} \mathrm{O}_{4}$ 纳 米粒子表面覆盖一层由凝胶-溶胶聚合而得到的间 苯二酚甲醛树脂保护壳 $\left(\mathrm{Fe}_{3} \mathrm{O}_{4} @ \mathrm{RF}\right)$ 。然后，通过己 烷溶胀的 $\mathrm{CTAB}$ 和 TEOS 复合物共自组装在 $\mathrm{Fe}_{3} \mathrm{O}_{4} @ \mathrm{RF}$
表面，形成 $\mathrm{Fe}_{3} \mathrm{O}_{4} @ \mathrm{RF} @ \mathrm{DFNS}$ 。用3-缩水甘油醚氧 基丙基三甲氧基硅烷(GLYMO)修饰后，胰蛋白可以 通过氨基酸和 GLYMO 中的环氧基反应，从而固定 在磁性复合纳米粒子的孔道中。所得复合纳米粒子 催化剂对小分子蛋白质展现了出色的尺寸选择性和 稳定性。

Yang 等 ${ }^{[47]}$ 将 $9 \mathrm{~nm}$ 的 $\mathrm{Fe}_{3} \mathrm{O}_{4}$ 纳米晶体嵌入 $\mathrm{KCC}-1$ 纤维孔道中, 生成具有磁性的纳米复合结构。严格来说, 该 $\mathrm{KCC}-1 / \mathrm{Fe}_{3} \mathrm{O}_{4}$ 结构不属于核一壳结构, 如图 6(b)所示。 将阿霉素(DOX) 负载于 $\mathrm{KCC}-1 / \mathrm{Fe}_{3} \mathrm{O}_{4}$ 之上, 所得 $\mathrm{KCC}-1 / \mathrm{Fe}_{3} \mathrm{O}_{4}$-DOX 复合结构可用来治疗癌症。

\subsection{2 非磁性核一壳结构}

$\mathrm{Wu}$ 等 ${ }^{[80]}$ 将沸石 TS-1 封入 KCC-1 内部, 形成 TS-1@KCC-1。不同于 KCC-1 的球形, TS-1@KCC-1 保留了 TS-1 的椭圆形貌(图 6(c))。TS-1@KCC-1 能 够很好地分散和稳定 $\mathrm{Rh}(\mathrm{OH})_{3}$, 形成的核-壳状 $(\mathrm{TS}-1) @ \mathrm{KCC}-1 / \mathrm{Rh}(\mathrm{OH})_{3}$ 能够一锅串联催化过氧化 氢氨和苯甲醛。当沸石 ZSM-5 作为中心核时, ZSM-5@KCC-1 为球状, 复合纳米粒子能够用一氧 化碳甲烷化 ${ }^{[107]}$, 乙苯脱氢和异丙基苯加氢裂解 ${ }^{[148]}$ 。 $\mathrm{Qu}$ 等 ${ }^{[134]}$ 成功制备了以实心二氧化硅球为核的 $\mathrm{SiO}_{2} @ \mathrm{DFNS}$ 核一壳结构(图6(d))。亲水性 $\mathrm{SiO}_{2} @ \mathrm{DFNS}$ 通过十八烷基三氯硅烷改性至疏水, 然后填充于高 效色谱分离柱，用来分离多肽类和蛋白质混合物。 树枝状纤维形结构使得分离效率高达 225000 plates $/ \mathrm{m}$ 。该课题组也使用核一壳状 $\mathrm{SiO}_{2} @$ DFNS 快 速分离小体积蛋白质、小分子、多肽类化合物和大 体积蛋白质 ${ }^{[77]}$ 。此外, $\mathrm{SiO}_{2} @ \mathrm{DFNS}$ 对芴类化合物的 分离效率高达 264531 plates $/ \mathrm{m}^{[69]}$ 。最近, 该研究小 组将金属有机骨架化合物 (Metal-organic Frameworks, MOFs)ZIF-8 修饰于 $\mathrm{SiO}_{2} @$ DFNS 孔道表面。 其对二甲苯同分异构体的分离效率达到 210000 plates $/ \mathrm{m}$, 复合结构 $\mathrm{SiO}_{2} @$ DFNS/ZIF-8 兼备 ZIF-8 的高选择性能和 DFNS 的高分离性能 ${ }^{[68]}$ 。Yu 等 ${ }^{[155]}$ 以 富勒烯 $\left(\mathrm{C}_{60}\right)$ 掺杂的二氧化硅为核, 树枝状纤维形二氧 化硅层作为壳, 核一壳状DFNS 纳米球 $\left(\mathrm{C}_{60} \& \mathrm{SiO}_{2} @ \mathrm{DFNS}\right)$ 能够负载单克隆抗体。富勒烯掺杂的硅核在紫外激 发下产生单分子氧气 $\left({ }^{1} \mathrm{O}_{2}\right)$, 能够作为光敏剂和生物 成像苂光剂。将上述复合纳米粒子递送于细胞内, 不仅能够用于细胞检测, 还能释放单克隆抗体来阻 止细胞调亡。

\section{2 空心结构}

在油水乳液中引入封端剂聚乙烯吡咯烷酮 (PVP), 于 $200^{\circ} \mathrm{C}$ 下可成功制备空心状 DFNS(图 6(e) $)^{[86]}$ 。 
空心状 DFNS 负载 CRL 所形成的 DFNS\&CRL 能够 高效活化庚酸和乙醇的酯化反应。采用囊泡自组装 方法，通过一锅法技术，可合成氨基均匀分布于骨 架的、呈正电性的空心状 DFNS ${ }^{[156]}$ 。所得 DFNS-NH 因而能够担载负电性蛋白质，展现了出色的细胞摄 取能力，更有效地将生物活性酶( $\beta$-半乳糖苷酶) 传 送至 CHO-K1 细胞 ${ }^{[156]}$ 。

\subsection{Janus 双面神结构 (不对称结构)}

Balkus 等 ${ }^{[121]}$ 将镁纳米粒子嵌入 DFNS 的孔道 中, 然后将金/钯涂覆在 DFNS/Mg 半球表面, 记为 $\mathrm{DFNS} / \mathrm{Mg} @ \mathrm{Au}$ 或 DFNS/Mg@Pt(图 6(f))。当不对称 结构浸入盐溶液, $\mathrm{Mg}$ 纳米粒子在 $\mathrm{Au}$ 或 $\mathrm{Pt}$ 的催化下 和水反应，产生的氢气 $\left(\mathrm{H}_{2}\right)$ 推动复合纳米粒子移动, 因而形成了能够应用在生物平台的运载纳米马 ${ }^{[121]}$ 。 Lee 等 ${ }^{[48]}$ 通过一锅法合成了羽毛球形不对称 DFNS, 中心放射褶皱形成于半球表面(图 6(g))。最近, $\mathrm{Yu}$ 等 ${ }^{[157]}$ 制备了类似羽毛球形的 DFNS，将它们称为“头一尾 形貌(Head-tail morphology)”。与对称结构或球形结
构相比较, 头-尾形 DFNS 作为强有力的辅佐剂能 够摄取和活化小鼠体内的抗原递呈细胞。该课题组 继进一步证明 “头-尾形貌”DFNS 具有尾长度-依赖 的溶血活性, 优异的血液相容性, 并且展示出对免 疫细胞较高程度的摄取能力 ${ }^{[158]}$ 。Zhang 等 ${ }^{[41]}$ 也制备 了伪-羽毛球形 DFNS, 只是树枝状纤维形二氧化硅 包裹在 RF 核半球表面，此制备法存在 TEOS 和 RF 的聚合竞争反应机制。

\section{4 蛋黄-蛋壳结构(摇铃形)}

赵东元等 ${ }^{[144]}$ 制备了 $\mathrm{Fe}_{3} \mathrm{O}_{4} @ \mathrm{RF} @$ DFNS 核-壳 双层结构, 当 $\mathrm{RF}$ 通过高温被烧去除后, 成功制备 了蛋黄-蛋壳形貌的 $\mathrm{Fe}_{3} \mathrm{O}_{4} @$ DFNS(图 6(h))。Zhang 等 ${ }^{[78]}$ 制备了 $\mathrm{Fe}_{3} \mathrm{O}_{4} @ \mathrm{SiO}_{2} @ \mathrm{RF} @ \mathrm{DFNS}$ 复合结构, 经过高 温羟烧处理, RF 树脂和 DFNS 结构中的 CTAB 模板 剂被去除, 形成了磁性蛋黄-蛋壳结构。氨基功能化 后，磁性蛋黄-蛋壳结构复合纳米粒子能够通过戊 二醛将 CRL 固定在其分层结构。15 次磁分离实验 后, CRL 活性依旧保持不变。

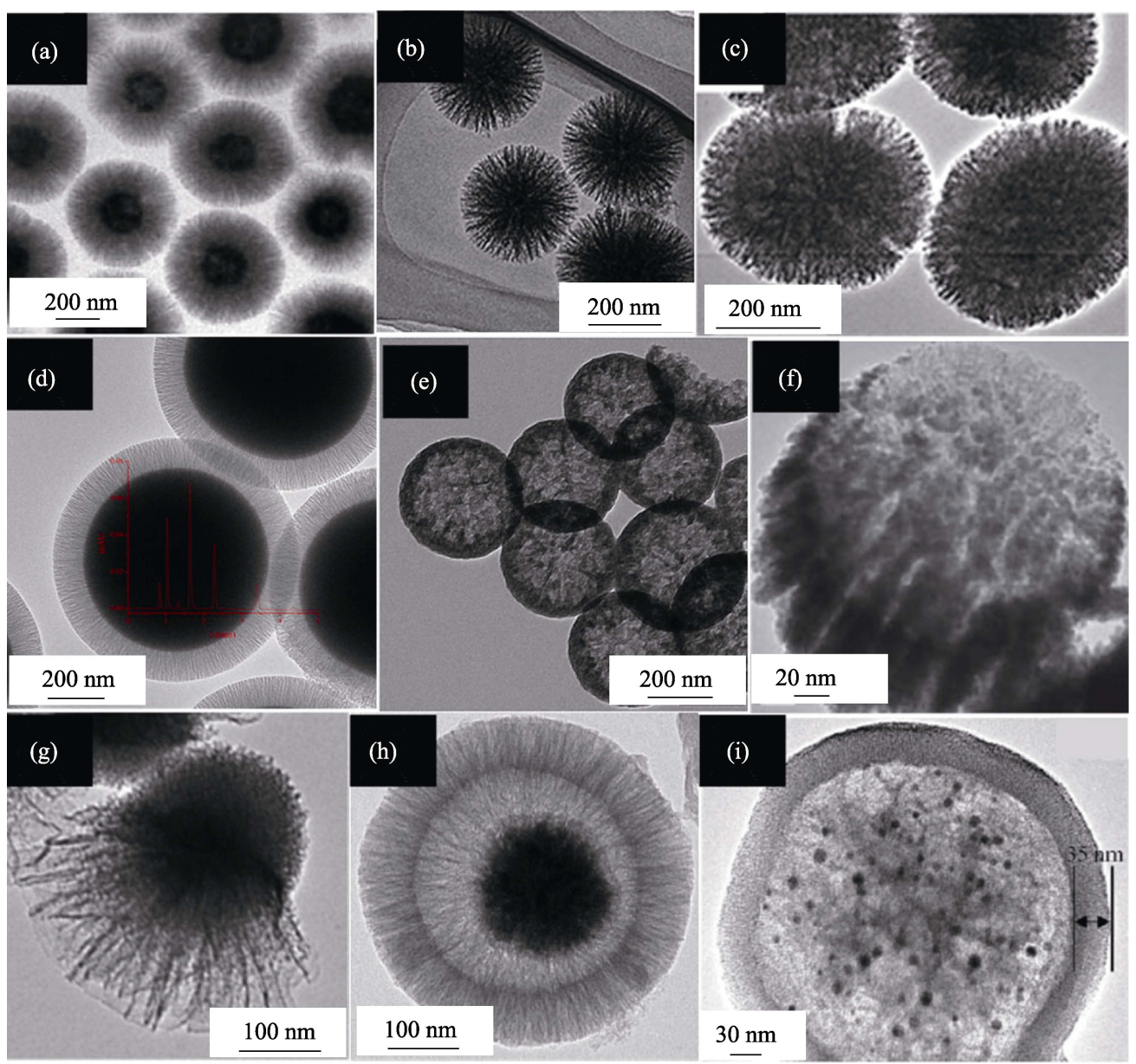

图 6 不同结构 DFNS 类复合纳米粒子的 TEM 照片

Fig. 6 TEM images of nanoparticles with diverse morphologies developed by means of DFNS reaction systems (a) Core-shelled $\mathrm{Fe}_{3} \mathrm{O}_{4} @ \mathrm{SiO}_{2} @ \mathrm{KCC}-1^{[8]}$; (b) $\mathrm{Fe}_{3} \mathrm{O}_{4} / \mathrm{DFNS}$ composites ${ }^{[47]}$; (c) Core-shelled TS-1@KCC-1 ${ }^{[80]}$;

(d) Core-shelled $\mathrm{SiO}_{2} @$ DFNS $^{[134]}$; (e) Hollow DFNS ${ }^{[86]}$; (f) Janus DFNS/Mg@A ${ }^{[121]}$; (g) Shuttlecock-shaped DFNS Au@Mg-DFNS ${ }^{[48]}$; (h) Yolk-shelled $\mathrm{Fe}_{3} \mathrm{O}_{4} @$ DFNS $^{[144]}$; (i) Yolk-shelled DFNS/Pt@MSS ${ }^{[159]}$ 


\section{5 其他特殊结构}

最近，杜至等 ${ }^{[159]}$ 制备了一种极其稳定的蛋黄蛋壳形异质催化剂, 用来催化还原对硝基苯酚。不 同于上述蛋黄-蛋壳结构的纳米粒子, 树枝状纤维 形壳结构在纳米粒子外部。此处, 树枝状纤维形二 氧化硅纳米球作为蛋黄结构, 而介孔二氧化硅层作 为保护壳(Mesoporous Silica Shell, MSS)。首先, 将 铂纳米颗粒载入 DFNS 核, 形成异质结构的 DFNS/Pt 蛋黄。然后，在其表面分别覆盖 RF 树脂和介孔硅壳， 形成 DFNS/Pt@RF@MSS。最后, 通过高温衫烧去 除 RF 有机树脂, 形成蛋黄-蛋壳形 DFNS/Pt@MSS (图 6(i))。该复合催化剂能够出色地催化一氧化碳氧化 和环化反应 ${ }^{[159]} 。 \mathrm{Li}$ 等 ${ }^{\left[{ }^{[1]}\right.}$ 以球形 DFNS 为核, 在其中 心辐射状孔道负载 $\mathrm{CdTe}$ 量子点后, 于表面制备了 一层介孔二氧化硅层。所得 DFNS/CdTe@MSS 具有 出色的稳定性和 MSS 壳厚度相关的细胞毒性, 能够 用于苂光生物成像。Zhou 等 ${ }^{[160]}$ 使用 PVP 作为封端 剂, 调节环己烷-乙醇-水反应体系中的 CTAB 浓度, 成功制备了以 DFNS 为蛋黄的摇铃形复合纳米粒子。

\section{6 实时应用概述}

DFNS 具有独特的三维中心辐射树枝状纤维形 超结构, 粒子内表面具有高度可接触性(可进入性)。 尤其是 DFNS 结构骨架存在硅羟基 $(-\mathrm{SiOH})$, 有机官 能团能够通过化学嫁接法修饰于其表面。不同尺寸 的分子(如药物和生物大分子)以及功能性纳米粒子 (如贵金属和苂光量子点)等都能够通过共价键合、 静电作用或疏水作用被原位负载或者共负载, 所形 成的特定功能化 DFNS 作为载体/支撑体/支架/平台 富有应用前景(图 3(b))。

随着 DFNS 蓬勃式发展，实时更新其应用领域 范围是必要的, 尤其是前面所提三篇综述未涉及内 容。本文在介绍非应用领域时(如第 5 节的新兴结构), 讲解了部分最新应用进展。现将 2017 2018 年 DFNS 出现的其他新颖应用实例介绍如下。

\subsection{DFNS 相关的 Pickering 乳液}

$\mathrm{Qu}$ 等 ${ }^{[116]}$ 将表面活化的人造酶(Surface-active Artificial Enzymes, SAE)纳米粒子负载于 DFNS 孔 道, 所得 DFNS/SAE 加入水和十二烷的混合液, 超 声作用下形成 Pickering 乳液。其中, 制备了三种类 型的 SAE，包括多肽类、金属复合物类和脱氧核酶 类，分别用于实现具有催化性能的仿生脂肪酶活 性、磷酸二酷酶活性和过氧化氢酶活性。 $\mathrm{Wu}$ 等 ${ }^{[118]}$ 将尼罗红(Nile red)负载于聚异丙基丙烯酰胺(PNIPAM)
功能化的球形 DFNS, 其作为 Pickering 乳化剂。加 入 5(6)-羧基苂光素(CFDA)并紫外照射, $\mathrm{pH}$ 响应的 单体聚合形成杂化胶体状微反应器, 其对 $\mathrm{pH}$ 和温 度有双刺激响应，即在不同温度和 $\mathrm{pH}$ 条件下尼罗 红和 CFDA 能被选择性活化释放。通过 Pickering 微乳液方法, Jiang 等 ${ }^{[161]}$ 使用脂肪酶功能化的球形 DFNS(Lp-DFNS)构筑了具有磁性及较强酶催化作 用的生物催化微胶囊(Colloidosome)。该生物催化微 胶囊能够催化不同烷基链长度的脂肪酸和醇的酯化 反应，比单纯脂肪酶催化的体系具有更高的产率。 此外, 此微胶囊能够催化丙三醇的酯基转移反应, 从而获得丙三醇碳酸酯, 转化率为 $85.2 \%$ 。Yang 等[45] 结合二甲基十八烷基 [3-(三甲氧基硅基)丙基]氯化 铵修饰的球形 DFNS 和离子液体技术，通过 Pickering 乳液制备了用于十二烯烃多相加氢甲酰化 的新型催化剂。

\section{2 实时生物应用}

Zhang 等 ${ }^{[162]}$ 将球形 DFNS 用氨基硅烷、琥珀酰 酐、阿仑膦酸钠(ALN) 和铁络合剂二乙三胺戊乙酸 改性，形成以 ALN 为骨靶向配体的复合平台担载 $\mathrm{Gd}^{3+}$ 和地塞米松(DEX)。终产物能够提高碱性磷酸酶活 性，从而提升间叶干细胞的结节形成能力。 $\mathrm{Hu}$ 等 ${ }^{[163]}$ 将疏水性的量子点(Quantum Dots, QDs)负载于颈基 改性的 DFNS 纳米孔道, 在 DFNS/QDs 表面涂覆烷 基硅烷层, 形成的 DFNS/QDs 胶体粒子能够灵敏且 持久地免疫测定临床 $\mathrm{C}$ 反应蛋白样品(C-reaction protein)。Bein 等 ${ }^{[164]}$ 用氮杂-二苯并环辛炔 (DBCO)改性 颈基化的大孔开口球形 DFNS, 来引发无铜催化的 叠氮化合物配合点击化学反应(如 5-羧基四甲基罗 丹明叠氮化物, TAMRA)。终结构 DFNS-DBCOTAMRA 能够将 TAMRA 受控释放于子宫颈癌传代 细胞。Jonuleit 等 ${ }^{[165]}$ 将肿瘤坏死因子 TNF- $\alpha$ 封装于 聚乙烯亚胺和聚乙二醇 (PEI-PEG) 改性的球形 DFNS，然后将 DFNS-(PEI-PEG)/TNF- $\alpha$ 递送于不同 癌细胞和树枝状细胞中, 来诱导细胞凋亡。

\section{3 球形 DFNS 作为硬模板}

球形 DFNS 一个非常重要的应用是作为硬模板 (牺牲模板), 通过纳米铸造的方法, 来制备具有相 似结构或相反结构的功能粒子, 例如: 树枝状纤维

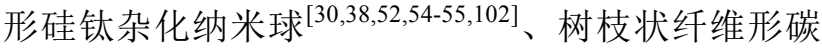
纳米球 ${ }^{[61,129,142]}$ 和树枝状纤维形碳氮杂化纳米球 ${ }^{[90,114]}$ 。 由于这一部分涉及内容较多, 将在后续的文章中详 细介绍。

\section{4 其他实时应用}

Costantini 等 ${ }^{[166]}$ 将 $\beta$-葡糖苷酶(Glucosidase, $\mathrm{BG}$ ) 
负载于 DFNS 的纳米褶皱孔道, 用来催化纤维二糖 水解, 四次循环可使得纤维二糖完全水解。

Polshettiwar 课题组 ${ }^{[71]}$ 将超细 $\mathrm{Au}$ 纳米粒子负载于 $\mathrm{NH}_{2}$-DFNS，所得催化剂不仅能高效催化氧化有机 硅烷生成硅醇, 还能实现硅烷醇解及醛类氯化。该 课题组 ${ }^{[29]}$ 最近将 DFNS\&Au 作为过氧化物酶, 相对于 天然的辣根过氧化物酶, 所得复合催化剂对四甲基 联苯胺催化具有很高酶催化活性。Polshettiwar 等 ${ }^{[27]}$ 在 DFNS 存在的螺吡喃溶液中观察到了对光反应变 色现象。具体为, 包含 DFNS 和螺吡喃的无色悬浊 液在紫外照射后变为蓝色。如果外加搅拌, 无色悬 浊液变为红色。研究者进一步用 DFNS 吸附螺吡喃 溶液中的部花青，生成的红色杂化材料在可见光照 射下显示出消极的对光反应变色现象 ${ }^{[28]}$ 。

\section{7 总结及展望}

本综述主要归纳总结了树枝状纤维形二氧化硅 纳米粒子的研究进展、结构特征、新兴结构及实时 应用领域等。对比分析了已开发 DFNS 合成方法的 优劣性，众多称谓的适用性、常用结构模型等。大 量研究成果表明: DFNS 独特的三维中心辐射状孔 道和多级孔结构, 使之极有可能替代传统二氧化硅 介孔材料(如 MCM-41 和 SBA-15) ${ }^{[75,102,138-139,167]}$, 成 为一种富有前景的平台/支撑体/载体。DFNS 已经推 动了一些新合成策略和应用领域的发展, 随着研究 的进一步深入, 今后的研究重点主要集中在以下三 个方面(图 7)。

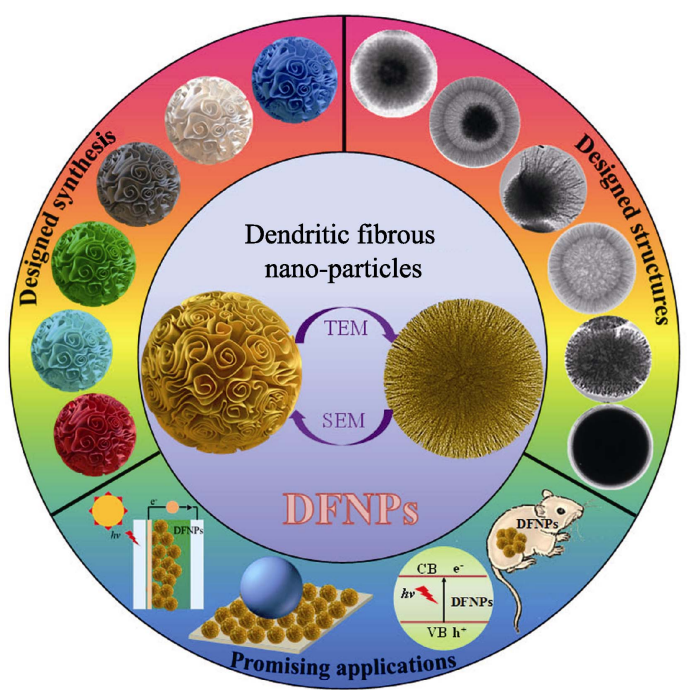

图 7 树枝状纤维形纳米粒子的设计合成、结构设计以及应 用前景示意图

Fig. 7 Schematic illustration of designed synthesis, designed structures, and promising applications for dendritic fibrous nano-particles

\section{1 设计合成}

DFNS 的合成方法启发众多研究课题组直接合 成其他本体元素基的树枝状纤维形纳米粒子 (Dendritic Fibrous Nano-particles, DFNPs), 如树枝 状纤维形活性生物玻璃纳米球 ${ }^{[122]}$ 。或通过引入新元 素对 DFNS 骨架掺杂改性, 如树枝状纤维形硅碳杂 化纳米球 ${ }^{[128]}$ 、树枝状纤维形硅铝杂化纳米球 ${ }^{[168]}$ 、 枝状纤维形硅铜铝杂化纳米球等 ${ }^{[104]}$ 。以上两种策略 是控制合成的重点，也是合成新型 DFNPs 的手段。

\section{2 结构设计}

基于不同元素组成的树枝状纤维形纳米粒子， 设计合成新颖结构或复杂多级结构依旧是该方向的 热点。例如, $Y u$ 等 ${ }^{[169]}$ 近期成功制备了空心状、双层 壳结构的树枝状纤维形有机一无机硅杂化纳米球。

\section{3 开拓新应用领域}

元素组成和独特结构共同决定了 DFNPs 的应 用前景。如上述空心双层壳结构的树枝状纤维形有 机一无机硅杂化纳米球能够应用于生物体进行药物 递送，进而达到肿瘤治疗的目的 ${ }^{[169]}$ 。一方面，有机一 无机硅杂化骨架较传统无机硅骨架更加稳定，也更 加容易被化学修饰进而负载治疗药物。另一方面, 空心状、双层壳、树枝状纤维形特殊结构可以控制 释放载药的速度，对细胞摄取也具有超强作用。

综上所述，以应用为目的，通过设计合成和结 构设计，树枝状纤维形纳米粒子这一学科展现出蓬 勃的生命力，大量工作亟需投入其中。

\section{参考文献:}

[1] LI W, LIU J, ZHAO D Y. Mesoporous materials for energy conversion and storage devices. Nature Reviews Materials, 2016, 1: 16023.

[2] SCH TH F, SCHMIDT W. Microporous and mesoporous materials. Advanced Engineering Materials, 2002, 4(5): 269-279.

[3] KRESGE C T, LEONOWICZ M E, ROTH W J, et al. Ordered mesoporous molecular sieves synthesized by a liquid-crystal template mechanism. Nature, 1992, 359: 710-712.

[4] ZHAO D Y, FENG J L, HUO Q S, et al. Triblock copolymer syntheses of mesoporous silica with periodic 50 to 300 angstrom pores. Science, 1998, 279(5350): 548-552.

[5] POLSHETTIWAR V, CHA D, ZHANG X X, et al. High-surface-area silica nanospheres (KCC-1) with a fibrous morphology. Angewandte Chemie International Edition, 2010, 49(50): 9652-9656.

[6] BAYAL N, SINGH B, SINGH R, et al. Size and fiber density controlled synthesis of fibrous nanosilica spheres (KCC-1). Scientific Reports, 2016, 6: 24888-1-11.

[7] FIHRI A, CHA D, BOUHRARA M, et al. Fibrous nano-silica (KCC-1)-supported palladium catalyst: Suzuki coupling reactions under sustainable conditions. ChemSusChem, 2012, 5(1): 85-89.

[8] YU K J, ZHANG X B, TONG H W, et al. Synthesis of fibrous 
monodisperse core-shell $\mathrm{Fe}_{3} \mathrm{O}_{4} / \mathrm{SiO}_{2} / \mathrm{KCC}-1$. Materials Letters, 2013, 106(9): 151-154.

[9] PENG H G, WANG D R, XU L, et al. One-pot synthesis of primary amides on bifunctional $\mathrm{Rh}(\mathrm{OH})_{x} / \mathrm{TS}-1 @ \mathrm{KCC}-1$ catalysts. Chinese Journal of Catalysis, 2013, 34(11): 2057-2065.

[10] BOUHRARA M, RANGA C, FIHRI A, et al. Nitridated fibrous silica (KCC-1) as a sustainable solid base nanocatalyst. ACS Sustainable Chemistry \& Engineering, 2013, 1(9): 1192-1199.

[11] HUANG X X, TAO Z M, PRASKAVICH J C, et al. Dendritic silica nanomaterials (KCC-1) with fibrous pore structure possess high DNA adsorption capacity and effectively deliver genes in vitro. Langmuir, 2014, 30(36): 10886-10898.

[12] FEBRIYANTI E, SUENDO V, MUKTI R R, et al. Further insight on the definite morphology and formation mechanism of mesoporous silica KCC-1. Langmuir, 2016, 32(23): 5802-5811.

[13] ALI Z, TIAN L, ZHANG B L, et al. Synthesis of paramagnetic dendritic silica nanomaterials with fibrous pore structure $\left(\mathrm{Fe}_{3} \mathrm{O}_{4} @ \mathrm{KCC}-1\right)$ and their application in immobilization of lipase from Candida rugosa with enhanced catalytic activity and stability. New Journal of Chemistry, 2017, 41(16): 8222-8231.

[14] FATAH N A A, TRIWAHYONO S, JALIL A A, et al. $n$-heptane isomerization over molybdenum supported on bicontinuous concentric lamellar silica KCC-1: influence of phosphorus and optimization using response surface methodology (RSM). Chemical Engineering Journal, 2017, 314: 650-659.

[15] DU X, HE J H. Fine-tuning of silica nanosphere structure by simple regulation of the volume ratio of cosolvents. Langmuir, 2010, 26(12): 10057-10062.

[16] DU X, QIAO S Z. Dendritic silica particles with center-radial pore channels: promising platforms for catalysis and biomedical applications. Small, 2015, 11(4): 392.

[17] DU X, SHI B Y, LIANG J, et al. Developing functionalized dendrimer-like silica nanoparticles with hierarchical pores as advanced delivery nanocarriers. Advanced Materials, 2013, 25(41): 5981-5985.

[18] DU X, XIONG L, DAI S, et al. Intracellular microenvironment responsive dendrimer-like mesoporous nanohybrids for traceable, effective, and safe gene delivery. Advanced Functional Materials, 2014, 24(48): 7627-7637.

[19] DU X, SHI B Y, TANG Y H, et al. Label-free dendrimer-like silica nanohybrids for traceable and controlled gene delivery. Biomaterials, 2014, 35(21): 5580-5590.

[20] DU X, XING Y, ZHOU M Y, et al. Broadband antireflective superhydrophilic antifogging nano-coatings based on three-layer system. Microporous and Mesoporous Materials, 2018, 255: 84-93.

[21] DU X, LI X Y, HUANG H W, et al. Dendrimer-like hybrid particles with tunable hierarchical pores. Nanoscale, 2015, 7(14): 6173-6184.

[22] SHEN D K, YANG J P, LI X M, et al. Biphase stratification approach to three-dimensional dendritic biodegradable mesoporous silica nanospheres. Nano Letters, 2014, 14(2): 923-932.

[23] DING X P, WANG Y B, HUANG Y D. Hydrothermal synthesis and characterization of fibrous silica nanospheres. Journal of Harbin Institute of Technology, 2018, 50(2): 116-121.

[24] DU X, ZHAO C X, HUANG H W, et al. Synthesis of dendrimerlike porous silica nanoparticles and their applications in advanced carrier. Progress in Chemistry, 2016, 28(8): 1131-1147.

[25] MAITY A, POLSHETTIWAR V. Dendritic fibrous nanosilica (DFNS) for catalysis, energy harvesting, $\mathrm{CO}_{2}$ mitigation, drug delivery and sensing. ChemSusChem, 2017, 10: 3866-3913.

[26] MAITY A, DAS A, SEN D, et al. Unraveling the formation mechanism of dendritic fibrous nanosilica. Langmuir, 2017,
33(48): 13774-13782.

[27] YAMAGUCHI T, MAITY A, POLSHETTIWAR V, et al. Photochromism of a spiropyran in the presence of a dendritic fibrous nanosilica; simultaneous photochemical reaction and adsorption. The Journal of Physical Chemistry A, 2017, 121(42): 8080-8085.

[28] YAMAGUCHI T, MAITY A, POLSHETTIWAR V, et al. Negative photochromism based on molecular diffusion between hydrophilic and hydrophobic particles in the solid state. Inorganic Chemistry, 2018, 57(7): 3671-3674.

[29] SINGH R, BELGAMWAR R, DHIMAN M, et al. Dendritic fibrous nano-silica supported gold nanoparticles as an artificial enzyme. Journal of Materials Chemistry B, 2018, 6(11): 1600-1604.

[30] BAYAL N, SINGH R, POLSHETTIWAR V. Nanostructured silica-titania hybrid using dendritic fibrous nanosilica as a photocatalyst. ChemSusChem, 2017, 10(10): 2182-2191.

[31] ZHANG S H, WEN L, YANG J P, et al. Facile fabrication of dendritic mesoporous $\mathrm{SiO}_{2} @ \mathrm{CdTe} @ \mathrm{SiO}_{2}$ fluorescent nanoparticles for bioimaging. Particle and Particle Systems Characterization, 2016, 33(5): 261-270.

[32] YANG J P, SHEN D K, WEI Y, et al. Monodisperse core-shell structured magnetic mesoporous aluminosilicate nanospheres with large dendritic mesochannels. Nano Research, 2015, 8(8): 2503-2514.

[33] DU X, HE J H. Hierarchically mesoporous silica nanoparticles: extraction, amino-functionalization, and their multipurpose potentials. Langmuir, 2011, 27(6): 2972-2979.

[34] DAI L L, ZHANG Q F, LI J H, et al. Dendrimer-like mesoporous silica nanoparticles as $\mathrm{pH}$-responsive nanocontainers for targeted drug delivery and bioimaging. ACS Applied Materials \& Interfaces, 2015, 7(13): 7357-7372.

[35] YU Y J, XING J L, PANG J L, et al. Facile synthesis of size controllable dendritic mesoporous silica nanoparticles. ACS Applied Materials \& Interfaces, 2014, 6(24): 22655-22665.

[36] XU Y, ZHU Y F, LI X L, et al. Investigation of dendritic mesoporous silica nanoparticles for cytosine phosphate guanosine oligodeoxynucleotide delivery. Materials Express, 2016, 6(2): 116-126.

[37] LEI C, XU C, NOUWENS A, et al. Ultrasensitive ELISA ${ }^{+}$enhanced by dendritic mesoporous silica nanoparticles. Journal of Materials Chemistry B, 2016, 4(29): 4975-4979.

[38] LIN C X C, XU C, YANG Y, et al. Dendritic mesoporous silicatitania nanospheres with enhanced photocatalytic activities. New Journal of Chemistry, 2017, 41: 8754-8760.

[39] WANG Y, NOR Y A, SONG H, et al. Small-sized and large-pore dendritic mesoporous silica nanoparticles enhance antimicrobial enzyme delivery. Journal of Materials Chemistry $B, 2016$, 4(15): 2646-2653.

[40] WANG J, WANG Y, LIU Q, et al. Rational design of multifunctional dendritic mesoporous silica nanoparticles to load curcumin and enhance efficacy for breast cancer therapy. ACS Applied Materials \& Interfaces, 2016, 8(40): 26511-26523.

[41] QU L L, HU H C, YU J Q, et al. High-yield synthesis of Janus dendritic mesoporous silica@resorcinol formaldehyde nanoparticles: a competing growth mechanism. Langmuir, 2017, 33(21): 5269-5274.

[42] LI Z X, ZHANG L L, TANG C, et al. Co-delivery of doxorubicin and survivin shRNA-expressing plasmid via microenvironment responsive dendritic mesoporous silica nanoparticles for synergistic cancer therapy. Pharmaceutical Research, 2017, 34(12): 2829-2841.

[43] HUANG M X, LIU L, WANG S G, et al. Dendritic mesoporous 
silica nanospheres synthesized by a novel dual-templating micelle system for the preparation of functional nanomaterials. Langmuir, 2017, 33(2): 519-526.

[44] XUE X L, LANG W Z, YAN X, et al. Dispersed vanadium in three-dimensional dendritic mesoporous silica nanospheres: active and stable catalysts for the oxidative dehydrogenation of propane in the presence of $\mathrm{CO}_{2}$. ACS Applied Materials \& Interfaces, 2017, 9(18): 15408-15423.

[45] TAO L, ZHONG M M, CHEN J, et al. Heterogeneous hydroformylation of long-chain alkenes in IL-in-oil Pickering emulsion. Green Chemistry, 2018, 20(1): 188-196.

[46] GAI S L, YANG P P, MA P A, et al. Uniform and size-tunable mesoporous silica with fibrous morphology for drug delivery. Dalton Transactions, 2012, 41(15): 4511-4516.

[47] GAI S L, YANG P P, MA P A, et al. Fibrous-structured magnetic and mesoporous $\mathrm{Fe}_{3} \mathrm{O}_{4} /$ silica microspheres: synthesis and intracellular doxorubicin delivery. Journal of Materials Chemistry, 2011, 21(41): 16420-16426.

[48] MOON D S, LEE J K. Formation of wrinkled silica mesostructures based on the phase behavior of pseudoternary systems. Langmuir, 2014, 30(51): 15574-15580.

[49] PANG J L, ZHOU G W, LIU R R, et al. Esterification of oleic acid with methanol by immobilized lipase on wrinkled silica nanoparticles with highly ordered, radially oriented mesochannels. Materials Science and Engineering C, 2016, 59: 35-42.

[50] JUNG D, KIM Y J, LEE J K. Novel strategy for maintenance of catalytic activity using wrinkled silica nanoparticle support in fischer-tropsch synthesis. Bulletin of the Korean Chemical Society, 2016, 37(3): 386-389.

[51] SHABIR J, GARKOTI C, SURABHI, et al. Development of amine functionalized wrinkled silica nanospheres and their application as efficient and recyclable solid base catalyst. Catalysis Letters, 2018, 148(1): 194-204.

[52] YOON C M, RYU J, YUN J, et al. Synthesis of hierarchical silica/ titania hollow nanoparticles and their enhanced electroresponsive activity. ACS Applied Materials \& Interfaces, 2018, 10(7): 6570-6579.

[53] WAN X J, ZHUANG L L, SHE B X, et al. In-situ reduction of monodisperse nanosilver on hierarchical wrinkled mesoporous silica with radial pore channels and its antibacterial performance. Materials Science and Engineering C, 2016, 65: 323-330.

[54] WANG Z J, BALKUS K J. Synthesis and modification of titanium containing wrinkled mesoporous silica for cyclohexene epoxidation. Microporous and Mesoporous Materials, 2017, 243: 76-84.

[55] WANG Z J, BALKUS K J. Liquid phase propylene oxidation with tert-butyl hydroperoxide over titanium containing wrinkled mesoporous silica. Catalysis Communications, 2017, 96: 15-18.

[56] HUANG X Y, TOWNLEY H. Knock-down of ELMO1 in paediatric rhabdomyosarcoma cells by nanoparticle mediated siRNA delivery. Nanobiomedicine, 2016, 3: 1-10.

[57] MUNAWEERA I, KONERU B, SHI Y, et al. Chemoradiotherapeutic wrinkled mesoporous silica nanoparticles for use in cancer therapy. APL Materials, 2014, 2(11): 123-128.

[58] MUNAWEERA I, SHI Y, KONERU B, et al. Nitric oxide- and cisplatin-releasing silica nanoparticles for use against non-small cell lung cancer. Journal of Inorganic Biochemistry, 2015, 153: 23-31.

[59] QIAN T T, LI J H, MIN X, et al. Radial-like mesoporous silica sphere: a promising new candidate of supporting material for storage of low-, middle-, and high-temperature heat. Energy, 2016, 112: 1074-1083.

[60] QIAN T T, YIN X P, LI J H, et al. Nano-TiO 2 decorated radial- like mesoporous silica: preparation, characterization, and adsorptionphotodegradation behavior. Journal of Materials Science and Technology, 2017, 33(11): 1314-1322.

[61] GUO D Y, CHEN X A, FANG Z P, et al. Hydrangea-like multi-scale carbon hollow submicron spheres with hierarchical pores for high performance supercapacitor electrodes. Electrochimica Acta, 2015, 176: 207-214.

[62] LEE S, CHOI K Y. Ethylene polymerization over metallocene catalysts supported on highly fibrous silica nanoparticles. Macromolecular Reaction Engineering, 2017, 11(1): 1600027-1-9.

[63] CHOI Y, YUN Y S, PARK H, et al. A facile approach for the preparation of tunable acid nano-catalysts with a hierarchically mesoporous structure. Chemical Communications, 2014, 50(57): 7652-7655.

[64] YANG H, LIAO S J, HUANG C, et al. Facile one-pot approach to the synthesis of spherical mesoporous silica nanoflowers with hierarchical pore structure. Applied Surface Science, 2014, 314(24): 7-14.

[65] YAMADA H, UJIIE H, URATA C, et al. A multifunctional role of trialkylbenzenes for the preparation of aqueous colloidal mesostructured/mesoporous silica nanoparticles with controlled pore size, particle diameter, and morphology. Nanoscale, 2015, 7(46): 19557-19567.

[66] YAMAMOTO E, KITAHARA M, TSUMURA T, et al. Preparation of size-controlled monodisperse colloidal mesoporous silica nanoparticles and fabrication of colloidal crystals. Chemistry of Materials, 2014, 26(9): 2927-2933.

[67] DU X, HE J H. Facile fabrication of hollow mesoporous silica nanospheres for superhydrophilic and visible/near-IR antireflection coatings. Chemistry-A European Journal, 2011, 17(29): 8165-8174.

[68] QU Q S, XUAN H, ZHANG K H, et al. Core-shell silica particles with dendritic pore channels impregnated with zeolite imidazolate framework-8 for high performance liquid chromatography separation. Journal of Chromatography A, 2017, 1505: 63-68.

[69] QU Q S, SI Y, XUAN H, et al. Dendritic core-shell silica spheres with large pore size for separation of biomolecules. Journal of Chromatography A, 2018, 1540: 31-37.

[70] TIAN J, YANG D J, WEN J G, et al. A stable rhodium singlesite catalyst encapsulated within dendritic mesoporous nanochannels. Nanoscale, 2018, 10(3): 1047-1055.

[71] DHIMAN M, CHALKE B, POLSHETTIWAR V. Organosilane oxidation with a half million turnover number using fibrous nanosilica supported ultrasmall nanoparticles and pseudo-single atoms of gold. Journal of Materials Chemistry A, 2017, 5(5): 1935-1940.

[72] RADHAKRISHNAN K, PANNEERSELVAM P, RAVIKUMAR A. A hybrid magnetic core-shell fibrous silica nanocomposite for a chemosensor-based highly effective fluorescent detection of Cu(II). RSC Advances, 2017, 7(72): 45824-45833.

[73] WANG Y F, ZHOU J J, ZHANG B L, et al. Fabrication and characterization of glutathione-imprinted polymers on fibrous $\mathrm{SiO}_{2}$ microspheres with high specific surface. Chemical Engineering Journal, 2017, 327: 932-940.

[74] FAN H T, LI B, SHI Z Z, et al. A fibrous morphology silica$\mathrm{CoFe}_{2} \mathrm{O}_{4}$ nanocarrier for anti-cancer drug delivery. Ceramics International, 2018, 44(2): 2345-2350.

[75] DENG X H, RIN R, TSENG J C, et al. Monodispersed mesoporous silica spheres supported $\mathrm{Co}_{3} \mathrm{O}_{4}$ as robust catalyst for oxygen evolution reaction. ChemCatChem, 2017, 9(22): 4238-4243.

[76] ALAMRI H, AL-SHAHRANI A, BOVERO E, et al. Selfcleaning superhydrophobic epoxy coating based on fibrous silica- 
coated iron oxide magnetic nanoparticles. Journal of Colloid and Interface Science, 2018, 513: 349-356.

[77] QU Q S, SI Y, XUAN H, et al. Synthesis of core-shell silica spheres with tunable pore diameters for HPLC. Materials Letters, 2018, 211: 40-42.

[78] ALI Z, TIAN L, ZHANG B L, et al. Synthesis of fibrous and non-fibrous mesoporous silica magnetic yolk-shell microspheres as recyclable supports for immobilization of candida rugosa lipase. Enzyme and Microbial Technology, 2017, 103: 42-52.

[79] AFZAL S, QUAN X, CHEN S, et al. Synthesis of manganese incorporated hierarchical mesoporous silica nanosphere with fibrous morphology by facile one-pot approach for efficient catalytic ozonation. Journal of Hazardous Materials, 2016, 318: 308-318.

[80] PENG H G, XU L, WU H L, et al. One-pot synthesis of benzamide over a robust tandem catalyst based on center radially fibrous silica encapsulated TS-1. Chemical Communications, 2013, 49(26): 2709-2711.

[81] ZHANG K, XU L L, JIANG J G, et al. Facile large-scale synthesis of monodisperse mesoporous silica nanospheres with tunable pore structure. Journal of the American Chemical Society, 2013, 135(7): 2427-2430.

[82] LIM S W, JANG H G, SIM H I, et al. Preparation of dandeliontype silica spheres and their application as catalyst supports. Journal of Porous Materials, 2014, 21(5): 797-809.

[83] MIN Y, YANG K G, LIANG Z, et al. Dandelion-like core-shell silica microspheres with hierarchical pores. RSC Advances, 2015, 5(33): 26269-26272.

[84] CHEN R K, YANG F D, XUE Y, et al. Polypyrrole confined in dendrimer-like silica nanoparticles for combined photothermal and chemotherapy of cancer. RSC Advances, 2016, 6(45): 38931-38942.

[85] ERNAWATI L, BALGIS R, OGI T, et al. Tunable synthesis of mesoporous silica particles with unique radially oriented pore structures from tetramethyl orthosilicate via oil-water emulsion process. Langmuir, 2017, 33(3): 783-790.

[86] WANG D D, LI X Y, LIU Z H, et al. Preparation of hollow silica nanospheres in $\mathrm{O} / \mathrm{W}$ microemulsion system by hydrothermal temperature changes. Solid State Sciences, 2017, 63: 62-69.

[87] WANG R L, HABIB E, ZHU X X. Synthesis of wrinkled mesoporous silica and its reinforcing effect for dental resin composites. Dental Materials, 2017, 33: 1139-1148.

[88] SING K S W. Reporting physisorption data for gas/solid systemswith special reference to the determination of surface area and porosity. Pure \& Applied Chemistry, 1985, 57(4): 603-619.

[89] DU X, HE J H. Amino-functionalized silica nanoparticles with center-radially hierarchical mesopores as ideal catalyst carriers. Nanoscale, 2011, 4(3): 852-859.

[90] BHUNIA M K, MELISSEN S, PARIDA M R, et al. Dendritic tip-on polytriazine-based carbon nitride photocatalyst with high hydrogen evolution activity. Chemistry of Materials, 2015, 27(24): 8237-8247.

[91] FR CHET J M J, TOMALIA D A, Dendrimers and Other Dendritic Polymers. New Jersey: John Wiley \& Sons, 2002: 3-40.

[92] POLSHETTIWAR V, THIVOLLE-CAZAT J, TAOUFIK M, et al. "Hydro-metathesis" of olefins: a catalytic reaction using a bifunctional single-site tantalum hydride catalyst supported on fibrous silica (KCC-1) nanospheres. Angewandte Chemie International Edition, 2011, 50(12): 2747-2751.

[93] PARK D S, YUN D, CHOI Y, et al. Effect of 3D open-pores on the dehydration of $n$-butanol to di- $n$-butyl ether (DNBE) over a supported heteropolyacid catalyst. Chemical Engineering Journal, 2013, 228(6): 889-895.
[94] HAMID M Y S, FIRMANSYAH M L, TRIWAHYONO S, et al. Oxygen vacancy-rich mesoporous silica KCC-1 for $\mathrm{CO}_{2}$ methanation. Applied Catalysis A General, 2017, 532: 86-94.

[95] DONG Z P, LE X D, DONG C X, et al. Ni@Pd core-shell nanoparticles modified fibrous silica nanospheres as highly efficient and recoverable catalyst for reduction of 4-nitrophenol and hydrodechlorination of 4-chlorophenol. Applied Catalysis B Environmental, 2015, 162(162): 372-380.

[96] LE X D, DONG Z P, LI X L, et al. Fibrous nano-silica supported palladium nanoparticles: an efficient catalyst for the reduction of 4-nitrophenol and hydrodechlorination of 4-chlorophenol under mild conditions. Catalysis Communications, 2015, 59: 21-25.

[97] LE X D, DONG Z P, LIU Y S, et al. Palladium nanoparticles immobilized on core-shell magnetic fibers as a highly efficient and recyclable heterogeneous catalyst for the reduction of 4-nitrophenol and Suzuki coupling reactions. Journal of Materials Chemistry A, 2014, 2(46): 19696-19706.

[98] QURESHI Z S, SARAWADE P B, ALBERT M, et al. Palladium nanoparticles supported on fibrous-structured silica nanospheres (KCC-1): an efficient and selective catalyst for the transfer hydrogenation of alkenes. ChemCatChem, 2015, 7(4): 635-642.

[99] QURESHI Z S, SARAWADE P B, HUSSAIN I, et al. Gold nanoparticles supported on fibrous silica nanospheres (KCC-1) as efficient heterogeneous catalysts for $\mathrm{CO}$ oxidation. ChemCatChem, 2016, 8: 1671-1678.

[100] SADEGHZADEH S M. A heteropolyacid-based ionic liquid immobilized onto fibrous nano-silica as an efficient catalyst for the synthesis of cyclic carbonate from carbon dioxide and epoxides. Green Chemistry, 2015, 17(5): 3059-3066.

[101] SIDDIQUI Z N, KHAN K, AHMED N. Nano fibrous silica sulphuric acid as an efficient catalyst for the synthesis of $\beta$-enaminone. Catalysis Letters, 2014, 144(4): 623-632.

[102] SINGH R, BAPAT R, QIN L J, et al. Atomic layer deposited (ALD) $\mathrm{TiO}_{2}$ on fibrous nano-silica (KCC-1) for photocatalysis: nanoparticle formation and size quantization effect. $\mathrm{ACS} C \mathrm{Ca}$ talysis, 2016, 6(5): 2770-2784.

[103] YANG H L, LI S W, ZHANG X Y, et al. Imidazolium ionic liquidmodified fibrous silica microspheres loaded with gold nanoparticles and their enhanced catalytic activity and reusability for the reduction of 4-nitrophenol. Journal of Materials Chemistry A, 2014, 2(2): 12060-12067.

[104] LAI L, ZHANG L L, HU C, et al. Enhanced Fenton-catalytic efficiency by highly accessible active sites on dandelion-like copper-aluminum-silica nanospheres for water purification. Journal of Materials Chemistry A, 2016, 4(22): 8610-8619.

[105] GAO J, KONG W X, ZHOU L Y, et al. Monodisperse core-shell magnetic organosilica nanoflowers with radial wrinkle for lipase immobilization. Chemical Engineering Journal, 2017, 309: 70-79.

[106] SHEN D K, CHEN L, YANG J P, et al. Ultradispersed palladium nanoparticles in three-dimensional dendritic mesoporous silica nanospheres: toward active and stable heterogeneous catalysts. ACS Applied Materials \& Interfaces, 2015, 7(31): $17450-17459$.

[107] TEH L P, TRIWAHYONO S, JALIL A A, et al. Fibrous silica mesoporous ZSM-5 for carbon monoxide methanation. Applied Catalysis A General, 2016, 523: 200-208.

[108] WU M, KONG L Y, WANG K W, et al. Enantioselective 1,2-reductions of $\beta$-trifluoromethylated- $\alpha, \beta$-unsaturated ketones to chiral allylic alcohols over organoruthenium-functionalized mesoporous silica nanospheres. Catalysis Science \& Technology, 
2015, 5(3): 1750-1757.

[109] LILLY THANKAMONY A S, LION C, SINGH B, et al. Insights into the catalytic activity of nitridated fibrous silica (KCC-1) nanocatalysts from ${ }^{15} \mathrm{~N}$ and ${ }^{29} \mathrm{Si}$ NMR spectroscopy enhanced by dynamic nuclear polarization. Angewandte Chemie International Edition, 2015, 54(7): 2190-2193

[110] DONG Z P, YU G Q, LE X D. Gold nanoparticle modified magnetic fibrous silica microspheres as a highly efficient and recyclable catalyst for the reduction of 4-nitrophenol. New Journal of Chemistry, 2015, 39(11): 8623-8629.

[111] DHIMAN M, CHALKE B, POLSHETTIWAR V. Efficient synthesis of monodisperse metal ( $\mathrm{Rh}, \mathrm{Ru}, \mathrm{Pd})$ nanoparticles supported on fibrous nanosilica (KCC-1) for catalysis. ACS Sustainable Chemistry \& Engineering, 2015, 3(12): 3224-3230.

[112] LE X D, DONG Z P, ZHANG W, et al. Fibrous nano-silica containing immobilized $\mathrm{Ni@Au} \mathrm{core-shell} \mathrm{nanoparticles:} \mathrm{a}$ highly active and reusable catalyst for the reduction of 4-nitrophenol and 2-nitroaniline. Journal of Molecular Catalysis A: Chemical, 2014, 395: 58-65.

[113] DONG Z P, LE X D, LI X L, et al. Silver nanoparticles immobilized on fibrous nano-silica as highly efficient and recyclable heterogeneous catalyst for reduction of 4-nitrophenol and 2-nitroaniline. Applied Catalysis B: Environmental, 2014, 158/159(1): 129-135.

[114] ZHANG J S, ZHANG M W, YANG C, et al. Nanospherical carbon nitride frameworks with sharp edges accelerating charge collection and separation at a soft photocatalytic interface. $A d$ vanced Materials, 2014, 26(24): 4121-4126.

[115] SADEGHZADEH S M, ZHIANI R, KHOOBI M, et al. Synthesis of 3-acyloxylindolines under mild conditions using tripolyphosphate-grafted KCC-1-NH 2 . Microporous and Mesoporous Materials, 2018, 257: 147-153.

[116] CHEN Z W, ZHAO C Q, JU E G, et al. Design of surface-active artificial enzyme particles to stabilize pickering emulsions for high-performance biphasic biocatalysis. Advanced Materials, 2016, 28(8): 1682-1688.

[117] MUNAWEERA I, HONG J, D'SOUZA A, et al. Novel wrinkled periodic mesoporous organosilica nanoparticles for hydrophobic anticancer drug delivery. Journal of Porous Materials, 2015, 22(1): 1-10.

[118] CONG Y, LI Q J, CHEN M, et al. Synthesis of dual-stimuliresponsive microcontainers with two payloads in different storage spaces for preprogrammable release. Angewandte Chemie International Edition, 2017, 56(13): 3552-3556.

[119] ABOUAITAH K E A, FARGHALI A A. Mesoporous silica materials in drug delivery system: $\mathrm{pH} /$ glutathione-responsive release of poorly water-soluble pro-drug quercetin from two and three-dimensional pore-structure nanoparticles. Journal of Nanomedicine and Nanotechnology, 2016, 7(2): 1000360-1-12.

[120] TSH A, LEE J H, LEE J J, et al. Mesoporous silica with fibrous morphology: a multifunctional core-shell platform for biomedical applications. Nanotechnology, 2013, 24(34): 345603-1-7.

[121] MUNAWEERA I, TRINH M, HONG J, et al. Chemically powered nanomotor as a delivery vehicle for biologically relevant payloads. Journal of Nanoscience and Nanotechnology, 2016, 16(9): 9063-9071.

[122] LI X, CHEN X F, MIAO G H, et al. Synthesis of radial mesoporous bioactive glass particles to deliver osteoactivin gene. Journal of Materials Chemistry B, 2014, 2(40): 7045-7054.

[123] XU C, YU M H, NOONAN O, et al. Core-cone structured monodispersed mesoporous silica nanoparticles with ultra-large cavity for protein delivery. Small, 2016, 11(44): 5949-5955.

[124] WU M Y, MENG Q S, CHEN Y, et al. Gene delivery: large-pore ultrasmall mesoporous organosilica nanoparticles: micelle/precursor co-templating assembly and nuclear-targeted gene delivery. Advanced Materials, 2015, 27(2): 215-222.

[125] SUN Z B, GUO D, ZHANG L, et al. Multifunctional fibrous silica composite with high optical sensing performance and effective removal ability toward $\mathrm{Hg}^{2+}$ ions. Journal of Materials Chemistry B, 2015, 3(16): 3201-3210.

[126] HUANG W Y, YU X, TANG J P, et al. Enhanced adsorption of phosphate by flower-like mesoporous silica spheres loaded with lanthanum. Microporous and Mesoporous Materials, 2015, 217: 225-232.

[127] XIE Y Y, WANG J, WANG M Z, et al. Fabrication of fibrous amidoxime-functionalized mesoporous silica microsphere and its selectively adsorption property for $\mathrm{Pb}^{2+}$ in aqueous solution. Journal of Hazardous Materials, 2015, 297: 66-73.

[128] YANG J P, CHEN W Y, SHEN D K, et al. Controllable fabrication of dendritic mesoporous silica-carbon nanospheres for anthracene removal. Journal of Materials Chemistry A, 2014, 2(29): 11045-11048.

[129] YU H X, ZHANG Q, DAHL M, et al. Dual-pore carbon shells for efficient removal of humic acid from water. Chemistry - $A$ European Journal, 2017, 23(64): 16249-16256.

[130] DU X, XING Y, LI X Y, et al. Broadband antireflective superhydrophobic self-cleaning coatings based on novel dendritic porous particles. RSC Advances, 2016, 6(10): 7864-7871.

[131] XING Y, DU X, LI X Y, et al. Tunable dendrimer-like porous silica nanospheres: effects of structures and stacking manners on surface wettability. Journal of Alloys and Compounds, 2018, 732: 70-79.

[132] PEREIRA C, ALVES C, MONTEIRO A, et al. Designing novel hybrid materials by one-pot co-condensation: from hydrophobic mesoporous silica nanoparticles to superamphiphobic cotton textiles. ACS Applied Materials \& Interfaces, 2011, 3(7): 2289-2299.

[133] LIU Y Y, LIU Q, YU H Y, et al. Polymer-modified fibrous mesoporous silica nanoparticles as coating material for open-tubular capillary electrochromatography. Journal of Chromatography A, 2017, 1499: 196-202.

[134] QU Q S, MIN Y, ZHANG L H, et al. Silica microspheres with fibrous shells: synthesis and application in HPLC. Analytical Chemistry, 2015, 87(19): 9631-9638.

[135] SUN Z B, LI H Z, GUO D, et al. A multifunctional magnetic core-shell fibrous silica sensing probe for highly sensitive detection and removal of $\mathrm{Zn}^{2+}$ from aqueous solution. Journal of Materials Chemistry C, 2015, 3(18): 4713-4722.

[136] CHEN P J, HU S H, HUNG W T, et al. Geometrical confinement of quantum dots in porous nanobeads with ultraefficient fluorescence for cell-specific targeting and bioimaging. Journal of Materials Chemistry, 2012, 22(22): 9568-9575.

[137] CHOI Y, KWAK H, HONG S. Quantification of arsenic(III) in aqueous media using a novel hybrid platform comprised of radially porous silica particles and a gold thin film. Analytical Methods, 2014, 6(17): 7054-7061.

[138] SINGH B, POLSHETTIWAR V. Design of $\mathrm{CO}_{2}$ sorbents using functionalized fibrous nanosilica (KCC-1): insights into the effect of the silica morphology (KCC-1 vs. MCM-41). Journal of Materials Chemistry A, 2016, 4(18): 7005-7019.

[139] PATIL U, FIHRI A, EMWAS A H, et al. Silicon oxynitrides of KCC-1, SBA-15 and MCM-41 for $\mathrm{CO}_{2}$ capture with excellent stability and regenerability. Chemical Science, 2012, 3(7): 2224-2229.

[140] YAMAMOTO E, MORI S, SHIMOJIMA A, et al. Fabrication of colloidal crystals composed of pore-expanded mesoporous 
silica nanoparticles prepared by a controlled growth method. Nanoscale, 2017, 9(7): 2464-2470.

[141] ZHANG H J, LI Z Y, XU P P, et al. A facile two step synthesis of novel chrysanthemum-like mesoporous silica nanoparticles for controlled pyrene release. Chemical Communications, 2010, 46(36): 6783-6785.

[142] PARK D S, YUN D, KIM T Y, et al. A mesoporous carbonsupported Pt nanocatalyst for the conversion of lignocellulose to sugar alcohols. ChemSusChem, 2013, 6(12): 2281-2289.

[143] KANG J S, LIM J, RHO W Y, et al. Wrinkled silica/titania nanoparticles with tunable interwrinkle distances for efficient utilization of photons in dye-sensitized solar cells. Scientific Reports, 2016, 6: 30829-1-14.

[144] YUE Q, LI J, LUO W, et al. An interface coassembly in biliquid phase: toward core-shell magnetic mesoporous silica microspheres with tunable pore size. Journal of the American Chemical Society, 2015, 137(41): 13282-13289.

[145] LI N, NIU D C, JIANG Y, et al. Morphology evolution and spatially selective functionalization of hierarchically porous silica nanospheres for improved multidrug delivery. Chemistry of Materials, 2017, 29(24): 10377-10385.

[146] TENG Z G, SU X D, ZHENG Y X, et al. A facile multi-interface transformation approach to monodisperse multiple-shelled periodic mesoporous organosilica hollow spheres. Journal of the American Chemical Society, 2015, 137(24): 7935-7944.

[147] YANG J P, SHEN D K, ZHOU L, et al. Spatially confined fabrication of core-shell gold nanocages@mesoporous silica for near-infrared controlled photothermal drug release. Chemistry of Materials, 2013, 25(15): 3030-3037.

[148] FIRMANSYAH M L, JALIL A A, TRIWAHYONO S, et al. Synthesis and characterization of fibrous silica ZSM-5 for cumene hydrocracking. Catalysis Science \& Technology, 2016(6): 5178-5182.

[149] DU X, HE J. Spherical silica micro/nanomaterials with hierarchical structures: synthesis and applications. Nanoscale, 2011, 3(10): 3984-4002.

[150] XU Z H, LI C X, KANG X J, et al. Synthesis of a multifunctional nanocomposite with magnetic, mesoporous, and near-IR absorption properties. Journal of Physical Chemistry C, 2010, 114(39): 16343-16350.

[151] LIU P C, YU Y J, PENG B, et al. A dual-templating strategy for the scale-up synthesis of dendritic mesoporous silica nanospheres. Green Chemistry, 2017, 19(23): 5575-5581.

[152] ZHANG A F, GU L, HOU K K, et al. Mesostructure-tunable and size-controllable hierarchical porous silica nanospheres synthesized by aldehyde-modified Stöber method. RSC $A d$ vances, 2015, 5(72): 58355-58362.

[153] SADEGHZADEH S M. A green approach for the synthesis of 2-oxazolidinones using gold(I) complex immobilized on KCC-1 as nanocatalyst at room temperature. Applied Organometallic Chemistry, 2016, 30(10): 835-842.

[154] SEO B, LEE C, YOO D, et al. A magnetically recoverable photocatalyst prepared by supporting $\mathrm{TiO}_{2}$ nanoparticles on a superparamagnetic iron oxide nanocluster core@fibrous silica shell nanocomposite. RSC Advances, 2017, 7(16): 9587-9595.

[155] ABBARAJU P L, YANG Y Y, YU M $\mathrm{H}$, et al. Core-shell-structured dendritic mesoporous silica nanoparticles for combined photodynamic therapy and antibody delivery. Chemistry-An Asian Journal, 2017, 12(13): 1465-1469.

[156] MEKA A K, ABBARAJU P L, SONG H, et al. A vesicle supraassembly approach to synthesize amine-functionalized hollow dendritic mesoporous silica nanospheres for protein delivery. Small, 2016, 12(37): 5169-5177.

[157] ABBARAJU P L, JAMBHRUNKAR M, YANG Y, et al. Asymmetric mesoporous silica nanoparticles as potent and safe immunoadjuvants provoke high immune responses. Chemical Communications, 2018, 54(16): 2020-2023.

[158] ABBARAJU P L, MEKA A K, SONG H, et al. Asymmetric silica nanoparticles with tunable head-tail structures enhance hemocompatibility and maturation of immune cells. Journal of the American Chemical Society, 2017, 139(18): 6321-6328.

[159] DU X, ZHAO C X, LUAN Y, et al. Dendritic porous yolk@ordered mesoporous shell structured heterogeneous nanocatalysts with enhanced stability. Journal of Materials Chemistry A, 2017, 5(40): 21560-21569.

[160] ZHENG Y J, WANG D D, LI Z K, et al. Laccase biosensor fabricated on flower-shaped yolk-shell $\mathrm{SiO}_{2}$ nanospheres for catechol detection. Colloids and Surfaces A: Physicochemical and Engineering Aspects, 2018, 538: 202-209.

[161] GAO J, WANG Y, DU Y J, et al. Construction of biocatalytic colloidosome using lipase-containing dendritic mesoporous silica nanospheres for enhanced enzyme catalysis. Chemical Engineering Journal, 2017, 317(315): 175-186.

[162] REN H, CHEN S, JIN Y, et al. A traceable and bone-targeted nanoassembly based on defect-related luminescent mesoporous silica for enhanced osteogenic differentiation. Journal of Materials Chemistry B, 2017, 5(8): 1585-1593.

[163] HUANG L, LIAO T, WANG J, et al. Brilliant pitaya-type silica colloids with central-radial and high-density quantum dots incorporation for ultrasensitive fluorescence immunoassays. $A d$ vanced Functional Materials, 2018, 28(4): 1705380-1-11.

[164] CHIU H Y, GÖßL D, HADDICK L, et al. Clickable multifunctional large-pore mesoporous silica nanoparticles as nanocarriers. Chemistry of Materials, 2018, 30(3): 644-654.

[165] KIENZLE A, KURCH S, SCHL DER J, et al. Dendritic mesoporous silica nanoparticles for $\mathrm{pH}$-stimuli-responsive drug delivery of TNF-Alpha. Advanced Healthcare Materials, 2017, 6(13): 1700012-1-9.

[166] CALIFANO V, SANNINO F, COSTANTINI A, et al. Wrinkled silica nanoparticles: efficient matrix for $\beta$-glucosidase immobilization. The Journal of Physical Chemistry C, 2018, 122(156): 8373-8379.

[167] XU J, ZHANG J Y, PENG H G, et al. Ag supported on mesostructured $\mathrm{SiO}_{2}$ with different morphologies for $\mathrm{CO}$ oxidation: on the inherent factors influencing the activity of Ag catalysts. Microporous and Mesoporous Materials, 2017, 242: 90-98.

[168] SHENG Y, ZENG H C. Monodisperse aluminosilicate spheres with tunable $\mathrm{Al} / \mathrm{Si}$ ratio and hierarchical macro-meso-microporous structure. ACS Applied Materials \& Interfaces, 2015, 7(24): 13578-13589.

[169] YANG Y N, LU Y, ABBARAJU P L, et al. Multi-shelled dendritic mesoporous organosilica hollow spheres: roles of composition and architecture in cancer immunotherapy. Angewandte Chemie International Edition, 2017, 56(29): 8446-8450. 